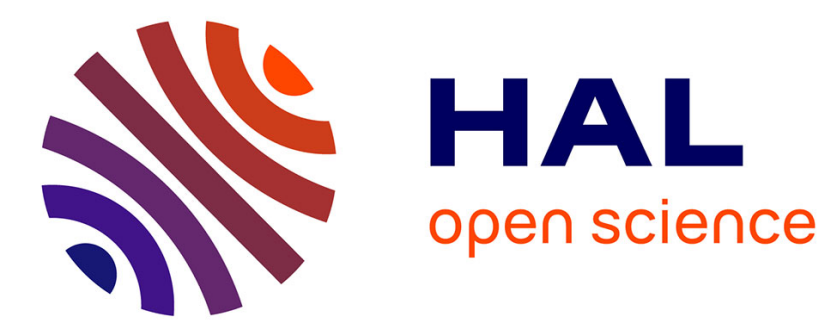

\title{
Second Kind Boundary Integral Equation for Multi-Subdomain Diffusion Problems
}

\author{
Xavier E Claeys, Ralf E Hiptmair, Elke E Spindler
}

\section{To cite this version:}

Xavier E Claeys, Ralf E Hiptmair, Elke E Spindler. Second Kind Boundary Integral Equation for Multi-Subdomain Diffusion Problems. Advances in Computational Mathematics, 2017, 43 (5), pp.26. hal-01427625

\section{HAL Id: hal-01427625 \\ https://hal.science/hal-01427625}

Submitted on 5 Jan 2017

HAL is a multi-disciplinary open access archive for the deposit and dissemination of scientific research documents, whether they are published or not. The documents may come from teaching and research institutions in France or abroad, or from public or private research centers.
L'archive ouverte pluridisciplinaire HAL, est destinée au dépôt et à la diffusion de documents scientifiques de niveau recherche, publiés ou non, émanant des établissements d'enseignement et de recherche français ou étrangers, des laboratoires publics ou privés. 


\title{
Second Kind Boundary Integral Equation for Multi-Subdomain Diffusion Problems
}

\author{
X.Claeys · R.Hiptmair • E.Spindler
}

Received: date / Accepted: date

\begin{abstract}
We consider isotropic scalar diffusion boundary value problems whose diffusion coefficients are piecewise constant with respect to a partition of space into Lipschitz subdomains. We allow so-called material junctions where three or more subdomains may abut. We derive a boundary integral equation of the second kind posed on the skeleton of the subdomain partition that involves, as unknown, only one trace function at each point of each interface. We prove the well-posedness of the corresponding boundary integral equations. We also report numerical tests for Galerkin boundary element discretisations, in which the new approach proves to be highly competitive compared to the well-established first kind direct single-trace boundary integral formulation. In particular, GMRES seems to enjoy fast convergence independent of the mesh resolution for the discrete second kind BIE.
\end{abstract}

Keywords second-order transmission problems · boundary integral equations $\cdot$ second kind single integral equations, · boundary element methods

Mathematics Subject Classification (2000) 65N12 - 65N38 - 65R20 • 35J05

Xavier Claeys

Sorbonne Universités, UPMC Univ. Paris 6 Laboratoire Jacques-Louis Lions, CNRS UMR 7598, and INRIA Paris, EPC Alpines, E-mail: claeys@ann.jussieu.fr

Ralf Hiptmair

Seminar of Applied Mathematics, ETH Zurich, E-mail: hiptmair@sam.math.ethz.ch

Elke Spindler

Seminar of Applied Mathematics, ETH Zurich, E-mail: elke.spindler@sam.math.ethz.ch 


\section{Introduction}

We consider the second-order diffusion problem

$$
\left\{\begin{array}{l}
-\operatorname{div}(\mu(\boldsymbol{x}) \nabla u)=0 \quad \text { in } \mathbb{R}^{3}, \\
\limsup _{|\boldsymbol{x}| \rightarrow \infty}|\boldsymbol{x}|\left|u_{\text {tot }}(\boldsymbol{x})-u_{\infty}(\boldsymbol{x})\right|<+\infty,
\end{array}\right.
$$

for a given excitation field $u_{\infty}$, harmonic on all of $\mathbb{R}^{3}$. We focus on piecewise constant real-valued diffusion coefficient functions $\mu \in L^{\infty}\left(\mathbb{R}^{3}\right)$. To describe them more precisely, we introduce a partition $\mathbb{R}^{d}=\cup_{j=0}^{n} \bar{\Omega}_{j}$, where each subdomain $\Omega_{j}, 1 \leq j \leq n$, is a bounded Lipschitz domain. Then we assume that $\mu$ is piecewise constant with respect to this partition, that is, for given numbers $\mu_{j}>0$,

$$
\mu_{\mid \Omega_{j}}=\mu_{j} \text { for all } j \in\{0, \ldots, n\} .
$$

Existence and uniqueness of solutions of (1) under suitable decay conditions are well established [30, Chapter 8]. Problems like (1) arise, for instance, in electrostatic models of dielectric bodies.

Boundary element methods based on reformulations of boundary value problems as boundary integral equations (BIE) are a popular class of computational techniques for problems like (1). A wealth of different BIE formulations are known for pure Dirichlet, Neumann, or mixed second-order scalar boundary value problems, and also transmission problems, that is, the case $n=1$ of (1), see [43, Chapter 3] or [22, Chapter 8]. A fundamental distinction is made between first kind and second kind BIEs. Their properties and that of related Galerkin boundary element methods are fairly well understood [43, Chapter 4], also for electromagnetic wave propagation [4] and elasticity [30, Chapter 10].

For the case $n>1$ of (1), the genuine multi-subdomain case, it is mainly first kind BIEs that have been proposed and investigated, see the seminal work [39] (based on [17]) and surveys in [10,9, Section 3 each]. Counterparts for time-harmonic electromagentic scattering, based on the Rumsey principle [42] have been known as PMCHWT BIEs for a long time [5,32,24] and their analysis has been accomplished in [3]. Polynomial Galerkin boundary element methods built on these formulations have to deal with ill-conditioned linear systems on fine meshes [43, Section 4.5] and, as a consequence, with slow convergence of iterative solvers. Preconditioning techniques drawing on ideas from domain decomposition like the Boundary Element Tearing and Interconnecting method (BETI) $[35,34,27,29]$, and Multi-Trace Formulations (MTF) [38, $37,8,9,7,25,10]$ are a remedy, but they entail rather complex algorithms.

Ill-conditioned Galerkin matrices are not an issue with second kind BIEs. In simple settings, $n=1$ for (1), and in the case of smooth geometries, the corresponding integral operators typically take the form of compact perturbations of the identity [22, Chapter 3] and, in conjunction with usual discretisation procedures (Galerkin, Nyström or collocation), yield well-conditioned matrices. 
Only recently the authors have proposed suitable integral equations of the second kind for genuine multi-subdomain problems. Initially, the focus was on the Helmholtz equation $-\Delta u-\kappa(\boldsymbol{x})^{2} u=f$ in $\mathbb{R}^{d}, d=2,3$ (with outgoing radiation condition), where $f$ is a source term, and the effective wave number $\kappa(\boldsymbol{x})$ is a constant $\kappa_{j}>0$ in each $\Omega_{j}$. Note that here the variable coefficient does not enter the principal part. For such wave propagation problems a socalled Single-Trace Formulation of the second kind (2nd-kind STF) has been proposed independently in [21] and [6,12]. A first extension of this approach was proposed in [11], where the authors considered the case of a propagation medium with impenetrable parts (homogeneous Dirichlet boundary condition imposed on one of the $\Omega_{j}$ 's). In [13] the idea was successfully applied to multisubdomain transmission problems for the time-harmonic Maxwell equations $\operatorname{curl}(\operatorname{curl} \mathbf{E})-\kappa(\boldsymbol{x})^{2} \mathbf{E}=0$. In this case the zero-order term in the differential operator does not represent a compact perturbation and new arguments are needed to derive a 2nd-kind STF. All details can be found in the PhD thesis [45].

Exploring 2nd-kind STF for Maxwell's equations taught us how to deal with variable coefficients in the principal part of the partial differential equations. This is exactly the situation we face with (1) and the present contribution elaborates the corresponding extension of the 2nd-kind STF. We arrive at integral equations reminiscent of so-called direct single integral equations as presented in [28].

The outline of this article is as follows. In Section 2 we describe precisely the geometry and the boundary value problem under consideration in the remainder of this article. In Section 3 we review basic definitions and results related to Sobolev spaces, trace operators, and the variational theory of the Laplace operator in free space. In Section 4 we introduce a functional framework well adapted to dealing with trace functions in a multi-subdomain context and, in the following section, we briefly review classical results on potential theory. In Section 6 we derive the new formulation for problems of the form (1), and we establish its well-posedness. This formulation then admits a variational formulation where trial functions are sought in single-trace spaces, and test functions are chosen in some complementary subspace. In Section 7 we rewrite this formulation so as to simplify the functional framework. With this reformulation, both trial and test functions are chosen in the same variational space consisting in trace functions defined on a cartesian product of interfaces. In this functional framework, each trace function belongs to a Sobolev space with (non trivial) fractional exponent. In Section 8, we show that the same formulation can still be considered in an even simpler framework based on square integrable traces. The final section presents 3D numerical experiments for the Galerkin boundary element discretisation of our new integral equations. The results highlight the competitiveness of our formulation compared to the more classical first kind approach. In particular, we always observe excellent conditioning of the Galerkin matrices generated by our new method. 
Remark 1 In spite of slight modifications due to the peculiarity of the Green's function of the Laplacian in two dimensions, our algorithms and the analysis can be easily adapted to problems set in $\mathbb{R}^{2}$. Nevertheless, we focus on the 3D setting for the sake of clarity.

\section{Setting of the problem}

Recall the partition of free space $\mathbb{R}^{3}:=\cup_{j=0}^{n} \bar{\Omega}_{j}$ where the $\Omega_{j}$ 's are Lipschitz domains. We assume that each $\Omega_{j}$ is bounded except $\Omega_{0}$. In the sequel we shall refer to the boundary of each subdomain by $\Gamma_{j}:=\partial \Omega_{j}$, and also set $\Gamma_{j, k}:=$ $\Gamma_{j} \cap \Gamma_{k}=\partial \Omega_{j} \cap \partial \Omega_{k}$ for reference to interfaces. The union of all interfaces, the skeleton, will be denoted by $\Sigma:=\cup_{j=0}^{n} \Gamma_{j}=\cup_{0 \leq j<k \leq n} \Gamma_{j, k}$. We are interested in solutions of (1), which should be understood in the weak sense, i.e., $u_{\text {tot }}$ belongs to the Sobolev space ${ }^{1} \mathrm{H}_{\mathrm{loc}}^{1}\left(\mathbb{R}^{3}\right)$ and satisfies $\int_{\mathbb{R}^{3}} \mu \nabla u_{\text {tot }} \nabla v d \boldsymbol{x}=0$ for all $v \in \mathrm{H}_{\text {comp }}^{1}\left(\mathbb{R}^{3}\right)$. Using the change of unknown $u=u_{\text {tot }}-u_{\infty}$, Problem (1) is equivalent to the transmission problem

$$
\begin{aligned}
& \left\{\begin{array}{l}
u \in \mathrm{H}_{\mathrm{loc}}^{1}\left(\mathbb{R}^{3}\right) \quad \text { with } \\
\Delta u=0 \quad \text { in } \Omega_{j}, \quad \forall j=0 \ldots n, \\
\lim \sup _{|\boldsymbol{x}| \rightarrow \infty}|\boldsymbol{x}||u(\boldsymbol{x})|<+\infty
\end{array}\right. \\
& \left\{\begin{array}{c}
\left.\mu_{j} \partial_{n_{j}} u\right|_{\Gamma_{j}}+\left.\mu_{k} \partial_{n_{k}} u\right|_{\Gamma_{k}}=-\left(\mu_{j} g_{j}+\mu_{k} g_{k}\right), \\
\left.u\right|_{\Gamma_{j}}-\left.u\right|_{\Gamma_{k}}=0 \quad \text { on } \Gamma_{j} \cap \Gamma_{k}, \forall j, k=0, \ldots, n,
\end{array}\right.
\end{aligned}
$$

where $\left.u\right|_{\Gamma_{j}}$ (resp. $\left.\partial_{n_{j}} u\right|_{\Gamma_{j}}:=\left.\mathbf{n}_{j} \cdot \nabla u\right|_{\Gamma_{j}}$ ) designates the traces of $u$ on $\Gamma_{j}$ (resp. the normal flux of $\nabla u$ at $\Gamma_{j}$ ) taken from the interior of $\Omega_{j}$, the vector field $\mathbf{n}_{j}$ is the normal to $\Gamma_{j}$ directed toward the exterior of $\Omega_{j}$, and the right hand side in (3b) is given by

$$
g_{j}:=\left.\partial_{n_{j}} u_{\infty}\right|_{\Gamma_{j}} \quad j=0 \ldots n .
$$

\section{Elementary function spaces}

To discuss the regularity properties of the solution to Problem (3), we need to introduce further notation regarding function spaces. We shall consider functions defined on volumic Lipschitz subsets $\omega \subset \mathbb{R}^{3}$, but also functions defined on the boundaries of such domains i.e. on Lipschitz manifolds. For these definitions and in terms of notations, we follow [30, Chap.3] as well as $[1,43]$ that can be consulted for more details.

1 Throughout we use standard notations for Sobolev spaces as found, for instance, in [30]. 
3.1 Volumic function spaces

Beside the usual (integer and fractional) Sobolev spaces $\mathrm{H}^{s}(\omega), s \in \mathbb{R}$, we write $\mathrm{H}^{s}(\operatorname{div}, \omega):=\left\{\mathbf{v} \in \mathrm{H}^{s}(\omega) \mid \operatorname{div}(\mathbf{v}) \in \mathrm{H}^{s}(\omega)\right\}$ with $\|\mathbf{v}\|_{\mathrm{H}^{s}(\operatorname{div}, \omega)}^{2}:=\|\mathbf{v}\|_{\mathrm{H}^{s}(\omega)}^{2}+$ $\|\operatorname{div}(\mathbf{v})\|_{\mathrm{H}^{s}(\omega)}^{2}$ (in the sequel $\mathrm{H}(\operatorname{div}, \omega)=\mathrm{H}^{s}(\operatorname{div}, \omega)$ with $s=0$ ), and use the space $\mathrm{H}^{1+s}(\Delta, \omega):=\left\{v \in \mathrm{H}^{s}(\omega) \mid \nabla v \in \mathrm{H}^{s}(\operatorname{div}, \omega)\right\}$ equipped with the corresponding natural norm

$$
\|v\|_{\mathrm{H}^{1+s}(\Delta, \omega)}^{2}=\|v\|_{\mathrm{H}^{1+s}(\omega)}^{2}+\|\Delta v\|_{\mathrm{H}^{s}(\omega)}^{2} .
$$

Recall that According to Theorem 3.30 and Theorem 3.33 of [30], for $s \in$ $(-1 / 2,+1 / 2)$, the space $\mathrm{H}^{-s}(\omega)$ is the topological dual to $\mathrm{H}^{+s}(\omega)$. With $\langle$, we denote the duality pairing between $\mathrm{H}^{s}(\omega)$ and $\mathrm{H}^{-s}(\omega)$.

\subsection{Trace spaces}

Recall [30, Lemma 3.35] that the Dirichlet trace $\left.\varphi \mapsto \varphi\right|_{\partial \omega}$ induces a continuous and surjective map sending $\mathrm{H}^{1+s}(\omega)$ onto $\mathrm{H}^{1 / 2+s}(\partial \omega)$ for $s \in(-1 / 2,1 / 2)$. We remind (see e.g. [43, Thm 2.7.7]) that the normal flux trace $\left.\mathbf{p} \mapsto \mathbf{n} \cdot \mathbf{p}\right|_{\partial \omega}$ can be extended by continuity to an operator mapping $\mathrm{H}_{\text {loc }}(\operatorname{div}, \bar{\omega})$ onto $\mathrm{H}^{-1 / 2}(\partial \omega)$. We shall actually need a sharper version of this continuity result.

\section{Lemma 1}

Consider any Lipschitz open set $\omega \subset \mathbb{R}^{3}$ with bounded boundary, denoting $\mathbf{n}$ the normal vector field to $\partial \omega$. For any $s \in(-1 / 2,+1 / 2)$ the normal flux operator $\left.\mathbf{p} \mapsto \mathbf{n} \cdot \mathbf{p}\right|_{\partial \omega}$ extends as a linear operator mapping continuously and surjectively $\mathrm{H}_{\mathrm{loc}}^{s}(\operatorname{div}, \bar{\omega})$ onto $\mathrm{H}^{s-1 / 2}(\partial \omega)$, and it is characterised by the Green's formula

$\int_{\omega} \mathbf{p} \cdot \nabla v+v \operatorname{div}(\mathbf{p}) d \boldsymbol{x}=\left\langle\left. v\right|_{\partial \omega},\left.\mathbf{n} \cdot \mathbf{p}\right|_{\partial \omega}\right\rangle \quad \forall \mathbf{p} \in \mathrm{H}_{\mathrm{loc}}^{s}(\operatorname{div}, \bar{\omega}), \forall v \in \mathrm{H}_{\mathrm{comp}}^{1-s}(\omega)$.

\section{Proof:}

Let $B \subset \mathbb{R}^{3}$ refer to a ball with radius sufficiently large to garantee that $\partial \omega \subset$ B. Define $\mathcal{O}:=\mathrm{B} \cap \omega$ so that $\mathcal{O}$ is bounded and $\partial \omega \subset \partial \mathcal{O}$. Fix $s \in$ $(-1 / 2,+1 / 2)$, and recall that there exists a continuous lifting operator $\mathcal{R}$ : $\mathrm{H}^{1 / 2-s}(\partial \omega) \rightarrow \mathrm{H}^{1-s}(\omega)$ such that $\left.\mathcal{R}(v)\right|_{\partial \omega}=v$ for all $v \in \mathrm{H}^{1 / 2-s}(\partial \omega)$, see for example [16, Lemma 4.2]. Using a cut-off function if necessary, one can consider in addition that $\operatorname{supp}\{\mathcal{R}(v)\} \subset \mathcal{O}$ for all $v \in \mathrm{H}^{1 / 2-s}(\partial \omega)$. Next, for any $\mathbf{p} \in \mathrm{H}_{\mathrm{loc}}^{s}(\operatorname{div}, \bar{\omega})$ define the functional $\varphi_{\mathbf{p}}$ by

$$
\varphi_{\mathbf{p}}(v):=\int_{\omega} \mathbf{p} \cdot \nabla \mathcal{R}(v)+\mathcal{R}(v) \operatorname{div}(\mathbf{p}) d \boldsymbol{x} \quad \forall v \in \mathrm{H}^{1 / 2-s}(\partial \omega) .
$$

Due to the regularity properties of $\mathbf{p}$, and the continuity of $\mathcal{R}$, the functional $\varphi_{\mathbf{p}}$ continuously maps $\mathrm{H}^{1 / 2-s}(\partial \omega)$ into $\mathbb{C}$ i.e. $\varphi_{\mathbf{p}} \in \mathrm{H}^{s-1 / 2}(\partial \omega)$, and it depends continuously on $\mathbf{p}$ in the norm of $\mathrm{H}^{s}(\operatorname{div}, \mathcal{O})$. Moreover it does not depend on the precise choice of $\mathcal{R}$ as long as $\left.\mathcal{R}(v)\right|_{\partial \omega}=v$, which is a direct consequence of 
Green's formula. Hence we set, as a definition, "n $\left.\cdot \mathbf{p}\right|_{\partial \omega} "=\varphi_{\mathbf{p}}$, which achieves the desired extension, so that Green's formula is satisfied by construction.

There only remains to prove the surjectivity of this normal flux operator. Pick an arbitrary $q \in \mathrm{H}^{s-1 / 2}(\partial \omega)$. According to Section 16 of [1], there exists a unique $u \in \mathrm{H}^{1+s}(\omega)$ solution to $-\Delta u+u=0$ in $\omega$ and $\left.\partial_{\mathbf{n}} u\right|_{\partial \omega}=q$. There only remains to take $\mathbf{p}=\nabla u \in \mathrm{H}^{s}(\operatorname{div}, \omega)$, so that $\left.\mathbf{n} \cdot \mathbf{p}\right|_{\partial \omega}=q$.

As an application of the preceding remarks, for each $s \in(-1 / 2,+1 / 2)$, every subdomain $\Omega_{j}$ supports continuous boundary trace operators $\gamma_{\mathrm{D}}^{j}: \mathrm{H}_{\mathrm{loc}}^{1+s}\left(\bar{\Omega}_{j}\right) \rightarrow$ $\mathrm{H}^{1 / 2+s}\left(\partial \Omega_{j}\right)$ and $\gamma_{\mathrm{N}}^{j}: \mathrm{H}_{\mathrm{loc}}^{1+s}\left(\Delta, \bar{\Omega}_{j}\right) \rightarrow \mathrm{H}^{-1 / 2+s}\left(\partial \Omega_{j}\right)$ (so-called Dirichlet and Neumann traces) uniquely defined by

$$
\gamma_{\mathrm{D}}^{j}(\varphi):=\left.\varphi\right|_{\partial \Omega_{j}} \quad \text { and } \quad \gamma_{\mathrm{N}}^{j}(\varphi):=\left.\mathbf{n}_{j} \cdot \nabla \varphi\right|_{\partial \Omega_{j}} \quad \forall \varphi \in \mathscr{C}^{\infty}\left(\mathbb{R}^{3}\right) .
$$

In the definition above, $\mathbf{n}_{j}$ is the unit vector field normal to $\partial \Omega_{j}$ pointing toward the exterior of $\Omega_{j}$. Define $\gamma_{\mathrm{D}, c}^{j}, \gamma_{\mathrm{N}, c}^{j}$ in the same manner as $\gamma_{\mathrm{D}}^{j}, \gamma_{\mathrm{N}}^{j}$ with traces taken from the exterior of $\Omega_{j}$. We shall also make use of mean values and jumps to these trace operators, defined as

$$
\left\{\gamma_{*}^{j}(u)\right\}:=\frac{1}{2}\left(\gamma_{*}^{j}(u)+\gamma_{*, c}^{j}(u)\right) \text { and }\left[\gamma_{*}^{j}(u)\right]:=\gamma_{*}^{j}(u)-\gamma_{*, c}^{j}(u) \text { for } *=\mathrm{D}, \mathrm{N} \text {. }
$$

\subsection{Regularity of solutions of diffusion problems}

In this paragraph, we would like to comment on the regularity of solutions to Problem (1) and (3). For this purpose we have to describe in more detail its natural variational setting. Define $\mathrm{W}^{1}\left(\mathbb{R}^{3}\right)$ as the completion of $\mathscr{C}_{\text {comp }}^{\infty}\left(\mathbb{R}^{3}\right)$ with respect to the following norm

$$
\|v\|_{\mathrm{W}^{1}\left(\mathbb{R}^{3}\right)}^{2}:=\int_{\mathbb{R}^{3}}|\nabla v|^{2}+\frac{|v(\boldsymbol{x})|^{2}}{1+|\boldsymbol{x}|^{2}} d \boldsymbol{x} .
$$

We shall also refer to the topological dual to $\mathrm{W}^{1}\left(\mathbb{R}^{3}\right)$ that we denote $\mathrm{W}^{-1}\left(\mathbb{R}^{3}\right):=$ $\mathrm{W}^{1}\left(\mathbb{R}^{3}\right)^{*}$, and write $\langle$,$\rangle for the duality pairing between \mathrm{W}^{1}\left(\mathbb{R}^{3}\right)$ and $\mathrm{W}^{-1}\left(\mathbb{R}^{3}\right)$. Given some $f \in \mathrm{W}^{-1}\left(\mathbb{R}^{3}\right)$ we will consider, for a short moment the variational problem:

$$
\begin{aligned}
& \text { Find } u \in \mathrm{W}^{1}\left(\mathbb{R}^{3}\right) \text { such that } \\
& \int_{\mathbb{R}^{3}} \mu \nabla u \nabla v d \boldsymbol{x}=\langle f, v\rangle \quad \forall v \in \mathrm{W}^{1}\left(\mathbb{R}^{3}\right) .
\end{aligned}
$$

It is a well known consequence of Hardy's inequality [23, Thm. 330] or [36], that this problem admits a unique solution. A natural question concerns the local regularity of its solution $u$ in the case where $f$ admits itself extra regularity, say $f \in \mathrm{H}_{\text {comp }}^{-1+s}\left(\mathbb{R}^{3}\right)$ with $s>0$, in spite of the coefficient $\mu$ admitting jumps (in particular $\mu$ is not Lipschitz). This may depend on the geometry of the partition, as was discussed in detail in $[31,33,40]$. For a general geometric configuration, an answer to this question was provided in [2, Thm.3.1]. Below is the statement of this result for the present context. 


\section{Theorem 1}

There exists $s_{\star} \in[0,1 / 2)$ that only depends on the partition $\mathbb{R}^{3}=\cup_{j=0}^{n} \bar{\Omega}_{j}$ such that for any $s \in\left[s_{\star}, 1 / 2\right]$, if $f \in \mathrm{H}_{\mathrm{comp}}^{-1 / 2-s}\left(\mathbb{R}^{3}\right)$, then the solution $u \in \mathrm{H}_{\mathrm{loc}}^{1}\left(\mathbb{R}^{3}\right)$ to Problem (5) actually belongs to $\mathrm{H}_{\mathrm{loc}}^{3 / 2-s}\left(\mathbb{R}^{3}\right)$. Morever the dependence is continuous: for any bounded set $\omega \subset \mathbb{R}^{3}$, there exists a constant $c_{\omega}>0$ independent of $u, f$ such that $\|u\|_{\mathrm{H}^{3 / 2-s}(\omega)} \leq c_{\omega}\|f\|_{\mathrm{H}^{-1 / 2-s}\left(\mathbb{R}^{3}\right)}$.

Here of course, we have reformulated this result so that it fits our notations, and did not state it in full generality. Let us point that, as underlined in [31,40], the smallest possible $s_{\star}$ in the previous theorem may be strictly greater than 0 for certain geometrical configurations. As a consequence of the continuity properties of the Neumann trace operator of Lemma 1, we deduce from this theorem the following result.

\section{Corollary 1}

Assume that $s_{\star} \in[0,1 / 2)$ is as in Theorem 1, and let $s \in\left[s_{\star}, 1 / 2\right]$. Then for any data $g_{j} \in \mathrm{H}^{-s}\left(\Gamma_{j}\right), j=0 \ldots n$, the unique $u \in \mathrm{W}^{1}\left(\mathbb{R}^{3}\right)$ satisfying

$$
\int_{\mathbb{R}^{3}} \mu \nabla u \nabla v d \boldsymbol{x}=\sum_{j=0}^{n} \mu_{j} \int_{\Gamma_{j}} g_{j} v d \sigma \quad \forall v \in \mathrm{W}^{1}\left(\mathbb{R}^{3}\right)
$$

actually satisfies $u \in \mathrm{H}_{\mathrm{loc}}^{3 / 2-s}\left(\mathbb{R}^{3}\right)$ with continuous dependency: for any bounded Lipschitz domain $\omega \subset \mathbb{R}^{3}$, there exists a constant $C_{\omega}>0$ independent of the $g_{j}$ 's and such that $\|u\|_{\mathrm{H}^{3 / 2-s}(\omega)} \leq C_{\omega} \sum_{j=0}^{n}\left\|g_{j}\right\|_{\mathrm{H}^{-s}\left(\Gamma_{j}\right)}$.

With the choice (4), Problem (6) is actually a variational formulation for (3). Since, in addition, $u_{\infty} \in \mathscr{C}^{\infty}\left(\mathbb{R}^{3}\right)$ due to local elliptic regularity, Corollary 1 is directly applicable to the problem under study here.

\section{Multi-subdomain trace spaces}

We aim for boundary integral equations set in natural trace spaces. The most fundamental trace space we can introduce is the Dirichlet/Neumann multitrace space [8, Sect. 2.1], given by the following Cartesian product:

$$
\begin{aligned}
& \mathbb{H}^{\sigma}(\Sigma):=\mathrm{H}^{\sigma}\left(\Gamma_{0}\right) \times \cdots \times \mathrm{H}^{\sigma}\left(\Gamma_{n}\right) \quad \text { for }|\sigma| \leq 1 / 2, \\
& \|\mathfrak{u}\|_{\mathbb{H}^{\sigma}(\Sigma)}:=\left(\left\|u_{0}\right\|_{\mathrm{H}^{\sigma}\left(\Gamma_{0}\right)}^{2}+\cdots+\left\|u_{n}\right\|_{\mathrm{H}^{\sigma}\left(\Gamma_{n}\right)}^{2}\right)^{\frac{1}{2}},
\end{aligned}
$$

for $\mathfrak{u}=\left(u_{0}, \ldots, u_{n}\right) \in \mathbb{H}^{\sigma}(\Sigma)$. Let us write $\langle,\rangle_{\Gamma_{j}}$ for the duality pairing between $\mathrm{H}^{\sigma}\left(\Gamma_{j}\right)$ and $\mathrm{H}^{-\sigma}\left(\Gamma_{j}\right)$. The spaces $\mathbb{H}^{+\sigma}(\Sigma)$ and $\mathbb{H}^{-\sigma}(\Sigma)$ are dual to each other with respect to the bilinear pairing

$$
\langle\langle\mathfrak{p}, \mathfrak{v}\rangle\rangle:=\sum_{j=0}^{n}\left\langle p_{j}, u_{j}\right\rangle_{\Gamma_{j}}, \quad \mathfrak{p}=\left(p_{j}\right)_{j=0}^{n} \in \mathbb{H}^{-\sigma}(\Sigma), \quad \mathfrak{v}=\left(v_{j}\right)_{j=0}^{n} \in \mathbb{H}^{+\sigma}(\Sigma) .
$$


For $\mathfrak{p} \in \mathbb{H}^{-\sigma}(\Sigma)$ and $\mathfrak{v} \in \mathbb{H}^{+\sigma}(\Sigma)$, we also adopt the convention $\langle\langle\mathfrak{v}, \mathfrak{p}\rangle\rangle:=$ $\langle\langle\mathfrak{p}, \mathfrak{v}\rangle\rangle$, which should not cause any further confusion. The bilinear form introduced above satisfies

$$
\inf _{\mathfrak{p} \in \mathbb{H}^{-\sigma}(\Sigma)} \sup _{\mathfrak{v} \in \mathbb{H}^{+\sigma}(\Sigma)} \frac{|\langle\langle\mathfrak{p}, \mathfrak{v}\rangle\rangle|}{\|\mathfrak{p}\|_{\mathbb{H}^{-\sigma}(\Sigma)}\|\mathfrak{v}\|_{\mathbb{H}^{+}(\Sigma)}}=1 .
$$

Single trace spaces. Next, as in [8, Sect. 2.2], [9, Sect. 3.1], we introduce the so-called single-trace space that consists of collections of traces that comply with transmission conditions. We first set, for $s \in(0,1)$

$$
\begin{aligned}
\mathbb{X}_{\mathrm{D}}^{s}(\Sigma):=\{\mathfrak{v} & =\left(v_{j}\right)_{j=0}^{n} \in \mathbb{H}^{s}(\Sigma) \mid \\
& \left.\exists v \in \mathrm{H}_{\mathrm{loc}}^{1 / 2+s}\left(\mathbb{R}^{3}\right), v_{j}=\gamma_{\mathrm{D}}^{j}(v), \forall j=0 \ldots n\right\} .
\end{aligned}
$$

It can be rather straightforwardly checked that $\mathbb{X}_{\mathrm{D}}^{s}(\Sigma)$ is a closed subspace of $\mathbb{H}^{s}(\Sigma)$. For $0<s<1$ and any $v \in \mathrm{L}_{\text {loc }}^{2}\left(\mathbb{R}^{3}\right)$ such that $\left.v\right|_{\Omega_{j}} \in \mathrm{H}_{\text {loc }}^{s+1 / 2}\left(\bar{\Omega}_{j}\right)$, we have $v \in \mathrm{H}_{\mathrm{loc}}^{s+1 / 2}\left(\mathbb{R}^{3}\right)$ if and only if the tuple of traces $\mathfrak{v}=\left(\gamma_{\mathrm{D}}^{j}(v)\right)_{j=0}^{n}$ belongs to $\mathbb{X}_{\mathrm{D}}^{s}(\Sigma)$, see e.g. $[1, \S 3.5]$. We define Neumann counterparts of these spaces by setting, for $s \in(0,1)$,

$$
\begin{aligned}
\mathbb{X}_{\mathrm{N}}^{-s}(\Sigma) & :=\left\{\mathfrak{p}=\left(p_{j}\right)_{j=0}^{n} \in \mathbb{H}^{-s}(\Sigma) \mid\right. \\
\quad \exists \mathbf{p} & \left.\in \mathrm{H}_{\mathrm{loc}}^{1 / 2-s}\left(\operatorname{div}, \mathbb{R}^{3}\right), p_{j}=\left.\mathbf{n}_{j} \cdot \mathbf{p}\right|_{\Gamma_{j}}, \forall j=0 \ldots n\right\} .
\end{aligned}
$$

Once again, since it is characterised by continuous constraints, this space is a closed subset of $\mathbb{H}^{-s}(\Sigma)$. The following lemma was proved in [6, Prop.2.1] in the case $s=1 / 2$. This proof can be readily adapted to the case of arbitrary $s \in(0,1)$ using the Green's formula of Lemma 1 above.

\section{Lemma 2}

For any $s \in(0,1)$, and $\mathfrak{u} \in \mathbb{H}^{+s}(\Sigma), \mathfrak{p} \in \mathbb{H}^{-s}(\Sigma)$ we have:

$$
\begin{aligned}
& \mathfrak{u} \in \mathbb{X}_{\mathrm{D}}^{+s}(\Sigma) \Longleftrightarrow\langle\langle\mathfrak{u}, \mathfrak{q}\rangle\rangle=0 \forall \mathfrak{q} \in \mathbb{X}_{\mathrm{N}}^{-s}(\Sigma), \\
& \mathfrak{p} \in \mathbb{X}_{\mathrm{N}}^{-s}(\Sigma) \Longleftrightarrow\langle\langle\mathfrak{v}, \mathfrak{p}\rangle\rangle=0 \forall \mathfrak{v} \in \mathbb{X}_{\mathrm{D}}^{+s}(\Sigma)
\end{aligned}
$$

One can provide an alternative, more algebraic characterisation of these spaces. Routine calculus in the sense of distributions using restrictions to interfaces shows that, for $0<s<1$, a tuple $\mathfrak{u}=\left(u_{j}\right)_{j=0}^{n} \in \mathbb{H}^{s}(\Sigma)$ satisfies

$$
\mathfrak{u} \in \mathbb{X}_{\mathrm{D}}^{+s}(\Sigma) \quad \Longleftrightarrow \quad u_{j}=u_{k} \text { on } \Gamma_{j} \cap \Gamma_{k}
$$

Similarly, for $0<s<1$, a tuple $\mathfrak{q}=\left(q_{j}\right)_{j=0}^{n} \in \mathbb{H}^{-s}(\Sigma)$ actually belongs to $\mathbb{X}_{\mathrm{N}}^{-s}(\Sigma)$ if we have $q_{j}=-q_{k}$ on $\Gamma_{j} \cap \Gamma_{k}$. 


\section{Potential theory}

In this paragraph, we shall remind the reader of well established results concerning the integral representation of solutions to homogeneous Helmholtz equation in Lipschitz domains. A detailed proof of the statements contained in the present paragraph can be found for example in [43, Chap.3]. Let

$$
\mathscr{G}(\boldsymbol{x}):=\frac{1}{4 \pi|\boldsymbol{x}|}
$$

refer to the Green's kernel associated to the Laplace operator. For each $\Omega_{j}$ and for any $v \in \mathrm{H}^{s+1 / 2}\left(\Gamma_{j}\right), q \in \mathrm{H}^{s-1 / 2}\left(\Gamma_{j}\right),|s| \leq 1 / 2$ and any $\boldsymbol{x} \in \mathbb{R}^{d} \backslash \Gamma_{j}$, define

$$
\begin{aligned}
\mathrm{SL}_{j}(q)(\boldsymbol{x}) & :=\int_{\Gamma_{j}} q(\boldsymbol{y}) \mathscr{G}(\boldsymbol{x}-\boldsymbol{y}) d \sigma(\boldsymbol{y}) \\
\mathrm{DL}_{j}(v)(\boldsymbol{x}) & :=\int_{\Gamma_{j}} v(\boldsymbol{y}) \mathbf{n}_{j}(\boldsymbol{y}) \cdot\left(\nabla \mathscr{G}_{\kappa}\right)(\boldsymbol{x}-\boldsymbol{y}) d \sigma(\boldsymbol{y}) .
\end{aligned}
$$

These operators are called single and double layer potentials. According to [16, Thm.1], The operator $\mathrm{SL}_{j}$ (resp. $\mathrm{DL}_{j}$ ) maps continuously $\mathrm{H}^{s-1 / 2}\left(\Gamma_{j}\right)$ (resp. $\left.\mathrm{H}^{s+1 / 2}\left(\Gamma_{j}\right)\right)$ into $\mathrm{H}_{\mathrm{loc}}^{1+s}\left(\Delta, \bar{\Omega}_{j}\right) \times \mathrm{H}_{\mathrm{loc}}^{1+s}\left(\Delta, \mathbb{R}^{d} \backslash \Omega_{j}\right)$ for $|s|<1 / 2$. As a consequence the following continuity properties hold.

\section{Proposition 1}

For any $j, k=0 \ldots n$, and any $s \in(-1 / 2,+1 / 2)$, the following are linear continuous maps:

$$
\begin{array}{r}
\gamma_{\mathrm{D}}^{k} \cdot \mathrm{DL}_{j}: \mathrm{H}^{+1 / 2+s}\left(\Gamma_{j}\right) \rightarrow \mathrm{H}^{+1 / 2+s}\left(\Gamma_{k}\right) \\
\gamma_{\mathrm{N}}^{k} \cdot \mathrm{DL}_{j}: \mathrm{H}^{+1 / 2+s}\left(\Gamma_{j}\right) \rightarrow \mathrm{H}^{-1 / 2+s}\left(\Gamma_{k}\right) \\
\gamma_{\mathrm{D}}^{k} \cdot \mathrm{SL}_{j}: \mathrm{H}^{-1 / 2+s}\left(\Gamma_{j}\right) \rightarrow \mathrm{H}^{+1 / 2+s}\left(\Gamma_{k}\right) \\
\gamma_{\mathrm{N}}^{k} \cdot \mathrm{SL}_{j}: \mathrm{H}^{-1 / 2+s}\left(\Gamma_{j}\right) \rightarrow \mathrm{H}^{-1 / 2+s}\left(\Gamma_{k}\right)
\end{array}
$$

These potential operators can be used to write a representation formula for solutions to homogeneous Laplace equations, see [43, Thm 3.1.6].

\section{Proposition 2}

For any $u \in \mathrm{H}_{\mathrm{loc}}^{1+s}\left(\Delta, \bar{\Omega}_{j}\right),|s|<1 / 2$, such that $\Delta u=0$ (and $\lim \sup _{|\boldsymbol{x}| \rightarrow \infty}|\boldsymbol{x} u(\boldsymbol{x})|<$ $+\infty$ in the case where $j=0)$, we have the representation formula

$$
\mathrm{SL}_{j}\left(\gamma_{\mathrm{N}}^{j}(u)\right)(\boldsymbol{x})+\mathrm{DL}_{j}\left(\gamma_{\mathrm{D}}^{j}(u)\right)(\boldsymbol{x})=u(\boldsymbol{x}) \mathbf{1}_{\Omega_{j}}(\boldsymbol{x})
$$

In this statement $\mathbf{1}_{\Omega_{j}}(\boldsymbol{x})=1$ if $\boldsymbol{x} \in \Omega_{j}$, and $\mathbf{1}_{\Omega_{j}}(\boldsymbol{x})=0$ otherwise. The potential operators $\mathrm{SL}_{j}, \mathrm{DL}_{j}$ also satisfy remarkable identities, known as jump formulas, describing their behaviour as $\boldsymbol{x}$ crosses $\Gamma_{j}=\partial \Omega_{j}$,

$$
\begin{aligned}
& {\left[\gamma_{\mathrm{D}}^{j}\right] \cdot \mathrm{DL}_{j}(v)=v\left[\gamma_{\mathrm{N}}^{j}\right] \cdot \mathrm{DL}_{j}(v)=0 \forall v \in \mathrm{H}^{s+\frac{1}{2}}\left(\Gamma_{j}\right),} \\
& {\left[\gamma_{\mathrm{D}}^{j}\right] \cdot \mathrm{SL}_{j}(q)=0\left[\gamma_{\mathrm{N}}^{j}\right] \cdot \mathrm{SL}_{j}(q)=q \forall q \in \mathrm{H}^{s-\frac{1}{2}}\left(\Gamma_{j}\right),}
\end{aligned}
$$


with $|s| \leq 1 / 2$. We will also need a remarkable property that arises when summing potential operators associated to all subdomains. This next result was proved in [6] for the case $s=1 / 2$. Adapting this proof to the case $s \in(0,1)$ does not raise any remarkable difficulty.

\section{Proposition 3}

For any $s \in(0,1)$, any $\left(p_{j}\right)_{j=0}^{n} \in \mathbb{X}_{\mathrm{N}}^{-s}(\Sigma)$ and any $\left(v_{j}\right)_{j=0}^{n} \in \mathbb{X}_{\mathrm{D}}^{+s}(\Sigma)$, and for all $\boldsymbol{x} \in \mathbb{R}^{3} \backslash \Sigma$ we have

$$
\sum_{j=0}^{n} \mathrm{SL}_{j}\left(p_{j}\right)(\boldsymbol{x})=0 \quad \text { and } \quad \sum_{j=0}^{n} \mathrm{DL}_{j}\left(v_{j}\right)(\boldsymbol{x})=0 .
$$

\section{Integral equation of the second kind}

In this section we show how two derive a boundary integral equation of the second kind for Problem (1). The unknowns will be related to the Neumann traces of the solution on the skeleton $\Sigma$. As a consequence, we start our analysis from the variational formulation (6) where the right hand side satisfies

$$
g_{j} \in \mathrm{H}^{-s}\left(\Gamma_{j}\right) \quad \forall s \in\left[s_{\star}, 1 / 2\right]
$$

where $s_{\star} \in[0,1 / 2)$ is as in Theorem 1 . For the solution $u \in \mathrm{W}^{1}\left(\mathbb{R}^{3}\right) \cap$ $\mathrm{H}_{\mathrm{loc}}^{3 / 2-s}\left(\mathbb{R}^{3}\right)$ of $(3)$, apply the representation formulas (12) in each subdomain $\Omega_{j}$, and sum for $j=0 \ldots n$. This yields

$$
u(\boldsymbol{x})=\sum_{j=0}^{n} \mathrm{SL}_{j}\left(\gamma_{\mathrm{N}}^{j}(u)\right)(\boldsymbol{x})+\sum_{j=0}^{n} \mathrm{DL}_{j}\left(\gamma_{\mathrm{D}}^{j}(u)\right)(\boldsymbol{x}), \quad \boldsymbol{x} \in \mathbb{R}^{3} \backslash \Sigma .
$$

Observe that, if $u$ is solution to (1), it satisfies the transmission conditions (3b) implying that $\left(\gamma_{\mathrm{D}}^{j}(u)\right)_{j=0}^{n} \in \mathbb{X}_{\mathrm{D}}^{1-s}(\Sigma)$. Hence, as a direct consequence of Proposition 3 the second term in (14) has to vanish. Since $\left(\gamma_{N}^{j}\left(u_{\infty}\right)\right)_{j=0}^{n} \in$ $\mathbb{X}_{\mathrm{N}}^{-s}(\Sigma)$ as $u_{\infty} \in \mathrm{H}_{\text {loc }}^{2}\left(\mathbb{R}^{3}\right)$, applying Proposition 3 and taking the Neumann trace of (14) on each subdomain $\Omega_{k}, k=0, \ldots, n$, we obtain

$$
\gamma_{\mathrm{N}}^{k}\left(u+u_{\infty}\right)-\sum_{j=0}^{n} \gamma_{\mathrm{N}}^{k} \cdot \mathrm{SL}_{j}\left(\gamma_{\mathrm{N}}^{j}\left(u+u_{\infty}\right)\right)=g_{k} \quad \text { with } \quad g_{k}:=\gamma_{\mathrm{N}}^{k}\left(u_{\infty}\right)
$$

Put this system in a matrix form, and consider the normal flux trace $\mathfrak{p}=$ $\left(\mu_{j} \gamma_{\mathrm{N}}^{j}\left(u+u_{\infty}\right)\right)_{j=0}^{n}$ as unkown. Taking account of the second transmission condition in $(3 \mathrm{~b})$, this unkown tuple of traces must be sought in $\mathbb{X}_{\mathrm{N}}^{-s}(\Sigma)$. Setting $\mathfrak{g}_{\mathrm{N}}:=\left(g_{k}\right)_{k=0}^{n} \in \mathbb{H}^{-s}(\Sigma), s \in\left[s_{\star}, 1 / 2\right]$, these equations take the form

$$
\left\{\begin{array}{l}
\text { Find } \mathfrak{p} \in \mathbb{X}_{\mathrm{N}}^{-s}(\Sigma) \text { such that } \\
\left\langle\left\langle\left(\mathrm{Id}-\mathbf{M}_{\mathrm{N}}\right) \cdot \mathbf{I}_{1 / \mu}(\mathfrak{p}), \mathfrak{v}\right\rangle\right\rangle=\left\langle\left\langle\mathfrak{g}_{\mathrm{N}}, \mathfrak{v}\right\rangle\right\rangle \quad \forall \mathfrak{v} \in \mathbb{H}^{+s}(\Sigma)
\end{array}\right.
$$


where $s \in\left[s_{\star}, 1 / 2\right]$, and

$$
\begin{aligned}
& \left\langle\left\langle\mathbf{M}_{\mathrm{N}}(\mathfrak{p}), \mathfrak{v}\right\rangle\right\rangle:=\sum_{j=0}^{n} \sum_{k=0}^{n}\left\langle\gamma_{\mathrm{N}}^{k} \cdot \mathrm{SL}_{j}\left(p_{j}\right), v_{k}\right\rangle_{\Gamma_{k}}, \\
& \left\langle\left\langle\mathbf{I}_{1 / \mu}(\mathfrak{p}), \mathfrak{v}\right\rangle\right\rangle:=\sum_{j=0}^{n} \mu_{j}^{-1}\left\langle p_{j}, v_{j}\right\rangle_{\Gamma_{j}} .
\end{aligned}
$$

As a direct application of Proposition 1, we see that the operators (17) induce linear operators continuously mapping $\mathbb{H}^{-s}(\Sigma)$ into $\mathbb{H}^{-s}(\Sigma)$ for any $s \in(0,1)$.

\subsection{Well-posedness}

In this section, we determine the kernel and the range of the operator (Id $\left.\mathbf{M}_{\mathrm{N}}\right) \cdot \mathbf{I}_{1 / \mu}$. First of all, we have the following non-trivial result that describes the "jump" of $\mathbf{M}_{\mathrm{N}}(\mathfrak{p})$ across interfaces of $\Sigma$.

\section{Proposition 4}

For any $s \in(0,1)$, we have $\left\langle\left\langle\left(\operatorname{Id}-\mathbf{M}_{\mathrm{N}}\right) \mathfrak{p}, \mathfrak{v}\right\rangle\right\rangle=0 \forall \mathfrak{p} \in \mathbb{H}^{-s}(\Sigma), \forall \mathfrak{v} \in \mathbb{X}_{\mathrm{D}}^{+s}(\Sigma)$.

\section{Proof:}

Consider any $s \in(0,1)$ that will be fixed until the end of the proof, and pick an arbitrary $\mathfrak{p}=\left(p_{0}, \ldots, p_{n}\right) \in \mathbb{H}^{-s}(\Sigma)$. For each $j=0 \ldots n$, define $\phi_{j} \in \mathrm{L}_{\text {loc }}^{2}\left(\mathbb{R}^{3} \backslash \Omega_{j}\right)$ by $\phi_{j}(\boldsymbol{x}):=\nabla \mathrm{SL}_{j}\left(p_{j}\right)(\boldsymbol{x})$ for $\boldsymbol{x} \in \mathbb{R}^{3} \backslash \bar{\Omega}_{j}$. Actually $\operatorname{div}\left(\phi_{j}\right)=0$ in $\mathbb{R}^{3} \backslash \bar{\Omega}_{j}$ so $\phi_{j} \in \mathrm{H}_{\text {loc }}^{1 / 2-s}\left(\operatorname{div}, \mathbb{R}^{3} \backslash \Omega_{j}\right)$ according to Section 5 , see also [16, Thm.1]. Next let $\psi$ refer to an element of $\mathrm{H}^{1}\left(\Omega_{j}\right)$ satisfying

$$
\Delta \psi=0 \quad \text { in } \Omega_{j}, \quad \gamma_{\mathrm{N}}^{j}(\psi)=\left.\mathbf{n}_{j} \cdot \phi\right|_{\Gamma_{j}}=\gamma_{\mathrm{N}, c}^{j} \cdot \mathrm{SL}_{j}\left(p_{j}\right) \quad \text { on } \Gamma_{j} .
$$

According to [44, Thm.4], we have $\psi \in \mathrm{H}^{3 / 2-s}\left(\Delta, \Omega_{j}\right)$, since $\gamma_{\mathrm{N}, c}^{j} \cdot \mathrm{SL}_{j}\left(p_{j}\right)=$ $-p_{j}+\gamma_{\mathrm{N}}^{j} \cdot \mathrm{SL}_{j}\left(p_{j}\right) \in \mathrm{H}^{-s}\left(\Gamma_{j}\right)$ according to Proposition 1 and (13) above. Now let us extend $\phi_{j}$ to the interior of $\Omega_{j}$ by setting $\left.\phi_{j}\right|_{\Omega_{j}}:=\nabla \psi$. This garantees that $\phi_{j} \in \mathrm{H}_{\mathrm{loc}}^{1 / 2-s}\left(\operatorname{div}, \mathbb{R}^{3}\right)$ due to the continuity of $\mathbf{n}_{j} \cdot \phi_{j}$ across $\Gamma_{j}$. Since $\left.\mathbf{n}_{j} \cdot \phi_{j}\right|_{\Gamma_{j}}=\gamma_{\mathrm{N}, c}^{j} \cdot \mathrm{SL}_{j}\left(p_{j}\right)$, by Definition (9) we have

$$
\mathfrak{q}_{j}:=\left(q_{j}^{k}\right)_{k=0}^{n} \in \mathbb{X}_{\mathrm{N}}^{-s}(\Sigma) \quad \text { where }\left\{\begin{array}{l}
q_{j}^{k}=\gamma_{\mathrm{N}}^{k} \cdot \mathrm{SL}_{j}\left(p_{j}\right) \text { for } j \neq k, \\
q_{j}^{j}=\gamma_{\mathrm{N}, c}^{j} \cdot \mathrm{SL}_{j}\left(p_{j}\right) .
\end{array}\right.
$$

In particular we have $\gamma_{\mathrm{N}}^{k} \cdot \mathrm{SL}_{j}\left(p_{j}\right)=q_{j}^{k}+\delta_{j}^{k}\left[\gamma_{\mathrm{N}}^{j}\right] \cdot \mathrm{SL}_{j}\left(p_{j}\right)=q_{j}^{k}+\delta_{j}^{k} p_{j}$ for all $j, k$. Here $\delta_{j}^{k}$ refers to Kronecker's symbol: $\delta_{j}^{k}=0$ if $j \neq k, \delta_{j}^{j}=1$. Now take an arbitrary $\mathfrak{v}=\left(v_{j}\right)_{j=0}^{n} \in \mathbb{X}_{\mathrm{D}}^{s}(\Sigma)$. Replace $\gamma_{\mathrm{N}}^{k} \cdot \mathrm{SL}_{j}\left(p_{j}\right)$ by $q_{j}^{k}$ in the expression 
of $\mathbf{M}_{\mathrm{N}}$ given by (17). Since $\left[\gamma_{\mathrm{N}}^{j}\right] \cdot \mathrm{SL}_{j}\left(p_{j}\right)=p_{j}$, applying Definition (10) leads to the conclusion of the proof

$$
\begin{aligned}
\left\langle\left\langle\mathbf{M}_{\mathrm{N}}(\mathfrak{p}), \mathfrak{v}\right\rangle\right\rangle & =\sum_{j=0}^{n} \sum_{k=0}^{n}\left\langle\gamma_{\mathrm{N}}^{k} \cdot \mathrm{SL}_{j}\left(p_{j}\right), v_{k}\right\rangle_{\Gamma_{k}} \\
& =\sum_{j=0}^{n}\left\langle\left\langle\mathfrak{q}_{j}, \mathfrak{v}\right\rangle\right\rangle+\left\langle\left[\gamma_{\mathrm{N}}^{j}\right] \cdot \mathrm{SL}_{j}\left(p_{j}\right), v_{j}\right\rangle_{\Gamma_{j}} \\
& =\sum_{j=0}^{n}\left\langle p_{j}, v_{j}\right\rangle_{\Gamma_{j}}=\langle\langle\mathfrak{p}, \mathfrak{v}\rangle\rangle .
\end{aligned}
$$

Combining the previous result with Proposition 3, we see that $\mathbf{M}_{\mathrm{N}}\left(\mathrm{Id}-\mathbf{M}_{\mathrm{N}}\right)=$ 0 , i.e. this operator is a projector. In addition we clearly have $\mathbb{X}_{\mathrm{N}}^{-s}(\Sigma) \subset$ $\operatorname{ker}\left(\mathbf{M}_{\mathrm{N}}\right)$ according to Proposition 3. Reciprocally, if $\mathfrak{p} \in \mathbb{H}^{-s}(\Sigma)$ satisfies $\mathbf{M}_{\mathrm{N}}(\mathfrak{p})=0$, then we have $\mathfrak{p}=\left(\mathrm{Id}-\mathbf{M}_{\mathrm{N}}\right) \mathfrak{p} \in \mathbb{X}_{\mathrm{N}}^{-s}(\Sigma)$ by Lemma 2 . To summarise, we have obtained the following result.

\section{Corollary 2}

We have $\left(\mathbf{M}_{\mathrm{N}}\right)^{2}=\mathbf{M}_{\mathrm{N}}$. In addition, for any $\mathfrak{p} \in \mathbb{H}^{-s}(\Sigma), s \in(0,1)$, we have $\mathbf{M}_{\mathrm{N}}(\mathfrak{p})=0$, if and only if $\mathfrak{p} \in \mathbb{X}_{\mathrm{N}}^{-s}(\Sigma)$.

We assumed that the right hand side $\mathfrak{g}_{\mathrm{N}}$ in (16) belongs to $\mathbb{X}_{\mathrm{N}}^{-s}(\Sigma)$ for all $s \in\left[s_{\star}, 1 / 2\right]$. A consequence of Proposition 4 is thus that Equation (16) yields a trivial identity whenever $\mathfrak{v}$ is chosen in $\mathbb{X}_{\mathrm{D}}^{+s}(\Sigma)$. This is a motivation for introducing a closed subspace $\mathbb{Y}^{s}(\Sigma) \subset \mathbb{H}^{s}(\Sigma)$ satisfying the complement condition

$$
\mathbb{H}^{s}(\Sigma)=\mathbb{X}_{\mathrm{D}}^{s}(\Sigma) \oplus \mathbb{Y}^{s}(\Sigma) .
$$

Such a complement subspace exists since $\mathbb{H}^{s}(\Sigma)$ is an Hilbert space. With this intermediate notation, the boundary integral formulation (16) can then be recast as a variational problem with different trial and test space: for $s \in$ $\left[s_{\star}, 1 / 2\right]$,

$$
\left\{\begin{array}{l}
\text { find } \mathfrak{p} \in \mathbb{X}_{\mathrm{N}}^{-s}(\Sigma) \text { such that } \\
\left\langle\left\langle\left(\operatorname{Id}-\mathbf{M}_{\mathrm{N}}\right) \cdot \mathbf{I}_{1 / \mu}(\mathfrak{p}), \mathfrak{v}\right\rangle\right\rangle=\left\langle\left\langle\mathfrak{g}_{\mathrm{N}}, \mathfrak{v}\right\rangle\right\rangle \quad \forall \mathfrak{v} \in \mathbb{Y}^{+s}(\Sigma) .
\end{array}\right.
$$

The next result shows that this formulation is actually well-posed.

\section{Proposition 5}

Let $s_{\star} \in[0,1 / 2)$ be as in Theorem 1. Then the operator $\left(\mathrm{Id}-\mathbf{M}_{\mathrm{N}}\right) \cdot \mathbf{I}_{1 / \mu}$ isomorphically maps $\mathbb{X}_{\mathrm{N}}^{-s}(\Sigma)$ onto $\mathbb{X}_{\mathrm{N}}^{-s}(\Sigma)$ for each $s \in\left[s_{\star}, 1 / 2\right]$.

\section{Proof:}

Pick an arbitrary $s \in\left[s_{\star}, 1 / 2\right]$ that will remain fixed until the end of the proof. Proposition 4 combined with Lemma 2 shows that the range of $\left(\mathrm{Id}-\mathbf{M}_{\mathrm{N}}\right) \cdot \mathbf{I}_{1 / \mu}$ is systematically contained in $\mathbb{X}_{\mathrm{N}}^{-s}(\Sigma)$. Let us first show that $\mathbb{X}_{\mathrm{N}}^{-s}(\Sigma) \cap \operatorname{ker}\left(\left(\operatorname{Id}-\mathbf{M}_{\mathrm{N}}\right) \cdot \mathbf{I}_{1 / \mu}\right)=\{0\}$. Take an arbitrary $\mathfrak{p}=\left(p_{j}\right)_{j=0}^{n} \in \mathbb{X}_{\mathrm{N}}^{-s}(\Sigma)$ such that $\left(\mathrm{Id}-\mathbf{M}_{\mathrm{N}}\right) \cdot \mathbf{I}_{1 / \mu}(\mathfrak{p})=0$. Set

$$
\psi(\boldsymbol{x}):=\sum_{j=0}^{n} \mu_{j}^{-1} \mathrm{SL}_{j}\left(p_{j}\right)(\boldsymbol{x}) \quad \forall \boldsymbol{x} \in \mathbb{R}^{3} \backslash \Sigma .
$$


According to $\left[16\right.$, Thm.1] we have $\mathrm{SL}_{j}\left(p_{j}\right) \in \mathrm{H}_{\text {loc }}^{3 / 2-s}\left(\mathbb{R}^{3}\right)$ for all $j$, which implies that $\gamma_{\mathrm{D}}^{j}(\psi)-\gamma_{\mathrm{D}}^{k}(\psi)=0$ on $\Gamma_{j} \cap \Gamma_{k}$ for all $j, k$. According to the previous observations, we have

$$
-\Delta \psi=0 \quad \text { in } \Omega_{j}, \quad \gamma_{\mathrm{D}}^{j}(\psi)-\gamma_{\mathrm{D}}^{k}(\psi)=0 \quad \text { on } \Gamma_{j} \cap \Gamma_{k} \quad \forall j, k=0, \ldots n .
$$

In addition $\left(\mathrm{Id}-\mathbf{M}_{\mathrm{N}}\right) \cdot \mathbf{I}_{1 / \mu}(\mathfrak{p})=0$ which can be re-written $\mu_{j}^{-1} p_{j}=\gamma_{\mathrm{N}}^{j}(\psi)$ or $p_{j}=\mu_{j} \gamma_{\mathrm{N}}^{j}\left(\psi_{j}\right)$. From this we conclude that $\left(\mu_{j} \gamma_{\mathrm{N}}^{j}\left(\psi_{j}\right)\right)_{j=0}^{n}=\mathfrak{p} \in \mathbb{X}_{\mathrm{N}}^{-s}(\Sigma)$ and thus, according to the polarity property of Lemma $2,0=\sum_{j=0}^{n} \int_{\Gamma_{j}} \mu_{j} \gamma_{\mathrm{N}}^{j}(\psi) \gamma_{\mathrm{D}}^{j}(\bar{\psi}) d \sigma=$ $\sum_{j=0} \int_{\Omega_{j}} \mu_{j}|\nabla \psi|^{2} d \boldsymbol{x}$ which implies that $\nabla \psi=0$ over $\mathbb{R}^{3}$, and thus $p_{j}=$ $\mu_{j} \gamma_{\mathrm{N}}^{j}(\psi)=0$ for all $j=0 \ldots n$.

To prove the surjectivity, take an arbitrary $\mathfrak{r}=\left(r_{j}\right)_{j=0}^{n} \in \mathbb{X}_{\mathrm{N}}^{-s}(\Sigma)$. Define $\phi \in \mathrm{H}_{\mathrm{loc}}^{1}\left(\mathbb{R}^{3}\right)$ as the unique solution to

$$
\phi \in \mathrm{W}^{1}\left(\mathbb{R}^{3}\right), \quad \int_{\mathbb{R}^{3}} \mu \nabla \phi \cdot \nabla v d \boldsymbol{x}=\sum_{j=0}^{n} \mu_{j} \int_{\Gamma_{j}} r_{j} \gamma_{\mathrm{D}}^{j}(v) d \sigma \quad \forall v \in \mathrm{W}^{1}\left(\mathbb{R}^{3}\right) .
$$

According to Corollary 1 , we actually have $\phi \in \mathrm{H}_{\text {loc }}^{3 / 2-s}\left(\mathbb{R}^{3}\right)$. Define $\mathfrak{r}^{\prime}=\left(r_{j}^{\prime}\right)_{j=0}^{n}$ by $r_{j}^{\prime}:=\mu_{j}\left(r_{j}-\gamma_{\mathrm{N}}^{j}(\phi)\right)$. Applying a Green's formula in (21) and using density of $\mathrm{H}_{\text {comp }}^{1}\left(\mathbb{R}^{3}\right)$ into $\mathrm{H}_{\text {comp }}^{1 / 2+s}\left(\mathbb{R}^{3}\right)$ for $s<1 / 2$, we obtain $\sum_{j=0}^{n} \int_{\Gamma_{j}} r_{j}^{\prime} \gamma_{\mathrm{D}}^{j}(v) d \sigma=0$ for all $v \in \mathrm{H}_{\text {comp }}^{1 / 2+s}\left(\mathbb{R}^{3}\right)$. According to (9) and Lemma 2, this implies that $\mathfrak{r}^{\prime} \in \mathbb{X}_{\mathrm{N}}^{-s}(\Sigma)$. Next Proposition 2 shows that

$$
\gamma_{\mathrm{N}}^{k} \cdot \mathrm{DL}_{j}\left(\gamma_{\mathrm{D}}^{j}(\phi)\right)+\gamma_{\mathrm{N}}^{k} \cdot \mathrm{SL}_{j}\left(\gamma_{\mathrm{N}}^{j}(\phi)\right)= \begin{cases}0 & \text { for } j \neq k \\ \gamma_{\mathrm{N}}^{j}(\phi) \text { for } j=k\end{cases}
$$

Sum equations $(22)$ for $j=0 \ldots n$. The terms associated to the single layer potential $\mathrm{DL}_{j}$ cancel out, as a consequence of Proposition 3 , since $\left(\gamma_{\mathrm{D}}^{j}(\phi)\right)_{j=0}^{n} \in$ $\mathbb{X}_{\mathrm{D}}^{1-s}(\Sigma)$ since $\phi \in \mathrm{H}_{\text {loc }}^{3 / 2-s}\left(\mathbb{R}^{3}\right)$ by construction. Noting that $\left(\gamma_{\mathrm{N}}^{j}(\phi)\right)_{j=0}^{n}=$ $\mathfrak{r}-\mathbf{I}_{1 / \mu}\left(\mathfrak{r}^{\prime}\right)$, we are left with

$$
\mathfrak{r}-\mathbf{I}_{1 / \mu}\left(\mathfrak{r}^{\prime}\right)=\mathbf{M}_{\mathrm{N}} \cdot\left(\mathfrak{r}-\mathbf{I}_{1 / \mu}\left(\mathfrak{r}^{\prime}\right)\right) \quad \Rightarrow \quad\left(\mathrm{Id}-\mathbf{M}_{\mathrm{N}}\right) \cdot \mathbf{I}_{1 / \mu}\left(\mathfrak{r}^{\prime}\right)=\mathfrak{r}-\mathbf{M}_{\mathrm{N}}(\mathfrak{r}) .
$$

There only remains to observe that, according to Corollary 2 , we have $\mathbf{M}_{\mathrm{N}}(\mathfrak{r})=$ 0 , since $\mathfrak{r} \in \mathbb{X}_{\mathrm{N}}^{-s}(\Sigma)$. Moreover, as $\mathfrak{r}^{\prime} \in \mathbb{X}_{\mathrm{N}}^{-s}(\Sigma)$ by construction, this ends the proof.

\section{Reduction to interfaces}

In this section, we wish to rewrite Formulation (19) in a more explicit manner. We will need the following additional, yet mild, assumption concerning the geometrical setting. 
Assumption 71 For any pair $j, k \in\{0, \ldots n\}$, the interface $\Gamma_{j} \cap \Gamma_{k}$ is either empty, or it is a point, or a Lipschitz curve of strictly positive length, or it is a Lipschitz two dimensional manifold with Lipschitz boundary and strictly positive surface measure.

Note that, in the case of $j=k$ we have $\Gamma_{j} \cap \Gamma_{k}$ is Lipschitz manifold (with no boundary). In this assumption the length and surface measure are the intrinsic ones induced by the ambient volume Lebesgue measure. In the case where $\Gamma_{j} \cap \Gamma_{k}$ is a non trivial Lipschitz two dimensional manifold, we shall simply write "area $\left(\Gamma_{j} \cap \Gamma_{k}\right)>0$ ". In practice, the assumption above is systematically satisfied, e.g., whenever each $\Omega_{j}$ is a curvilinear polyhedron.

According to Theorem 3.33 and Theorem 3.40 of [30], with this assumption and in the case that $s \in(-1 / 2,+1 / 2)$, on the boundary of any subdomain we can decompose trace spaces as follows

If $|s|<1 / 2, \quad v \in \mathrm{H}^{s}\left(\Gamma_{j}\right) \Longleftrightarrow\left\{\begin{array}{l}\left.v\right|_{\Gamma_{j} \cap \Gamma_{k}} \in \mathrm{H}^{s}\left(\Gamma_{j} \cap \Gamma_{k}\right) \\ \forall k=0 \ldots n \text { such that area }\left(\Gamma_{j} \cap \Gamma_{k}\right)>0 .\end{array}\right.$

In other words, to guarantee a sufficient regularity of a trace function on $\Gamma_{j}$, it suffices to examine its regularity on each interface. It is important to note that (23) does not hold for $|s| \geq \pm 1 / 2$ and in particular not for $s= \pm 1 / 2$. Observation (23) leads us to introduce a decomposition of the skeleton into interfaces.

$$
\begin{aligned}
& \Sigma=\cup_{\mathrm{J} \in \mathfrak{I}} \bar{\Gamma}_{\mathrm{J}} \text { where } \quad \Gamma_{\mathrm{J}}:=\Gamma_{\mathrm{J}_{+}} \cap \Gamma_{\mathrm{J}_{-}} \text {with } \\
& \mathfrak{I}:=\left\{\mathrm{J}=\left(\mathrm{J}_{-}, \mathrm{J}_{+}\right) \in\{0, \ldots n\}^{2} \mid \mathrm{J}_{-}<\mathrm{J}_{+}\right. \text {and } \\
& \left.\Gamma_{\mathrm{J}_{+}} \cap \Gamma_{\mathrm{J}_{-}} \text {is a non-trivial Lipschitz manifold }\right\} .
\end{aligned}
$$

For any interface let us denote $\langle\cdot, \cdot\rangle_{\Gamma_{\mathrm{J}}}$ the duality pairing between $\mathrm{H}^{s}\left(\Gamma_{\mathrm{J}}\right)$ and $\mathrm{H}^{-s}\left(\Gamma_{\mathrm{J}}\right)$ for $|s|<1 / 2$. As a consequence of $(23)$, each $\langle\cdot, \cdot\rangle_{\Gamma_{j}}$ is naturally decomposed into a sum of such interface duality pairings. Now pick arbitrary $\mathfrak{u}=\left(u_{j}\right)_{j=0}^{n} \in \mathbb{H}^{+s}(\Sigma), \mathfrak{v}=\left(v_{j}\right)_{j=0}^{n} \in \mathbb{H}^{-s}(\Sigma)$, and let us rewrite $\langle\langle\mathfrak{u}, \mathfrak{v}\rangle\rangle$ according to decomposition (24). For each $s \in(-1 / 2,1 / 2)$, using the parallelogram identity, the global duality pairing decomposes as follows

$$
\begin{aligned}
\langle\langle\mathfrak{u}, \mathfrak{v}\rangle\rangle & =\sum_{j=0}^{n}\left\langle u_{j}, v_{j}\right\rangle_{\Gamma_{j}}=\sum_{\mathrm{J} \in \mathfrak{I}}\left\langle u_{\mathrm{J}_{+}}, v_{\mathrm{J}_{+}}\right\rangle_{\Gamma_{\mathrm{J}}}+\left\langle u_{\mathrm{J}_{-}}, v_{\mathrm{J}_{-}}\right\rangle_{\Gamma_{\mathrm{J}}} \\
& =\sum_{\mathrm{J} \in \mathfrak{I}} 2\left\langle\left\{u_{\mathrm{J}}\right\},\left\{v_{\mathrm{J}}\right\}\right\rangle_{\Gamma_{\mathrm{J}}}+\left\langle\left[u_{\mathrm{J}}\right],\left[v_{\mathrm{J}}\right]\right\rangle_{\Gamma_{\mathrm{J}}} / 2 \quad \forall \mathfrak{u} \in \mathbb{H}^{+s}(\Sigma), \forall \mathfrak{v} \in \mathbb{H}^{-s}(\Sigma) \\
& \text { setting }\left\{u_{\mathrm{J}}\right\}:=\left(u_{\mathrm{J}_{+}}+u_{\mathrm{J}_{-}}\right) / 2 \quad \text { and } \quad\left[u_{\mathrm{J}}\right]:=u_{\mathrm{J}_{+}}-u_{\mathrm{J}_{-}} .
\end{aligned}
$$

For an arbitrary $\mathfrak{u} \in \mathbb{H}^{-s}(\Sigma), 0<s<1 / 2$, we have $\left\{u_{\mathrm{J}}\right\}=0 \forall \mathrm{J} \in \mathfrak{I}$ if and only if $\mathfrak{u}=\left(u_{j}\right)_{j=0}^{n} \in \mathbb{X}_{\mathrm{N}}^{-s}(\Sigma)$. Similarly, for any $\mathfrak{u} \in \mathbb{H}^{+s}(\Sigma), 0<s<1 / 2$ we have $\left[u_{\mathrm{J}}\right]=0 \forall \mathrm{J} \in \mathfrak{I}$ if and only if $\mathfrak{u}=\left(u_{j}\right)_{j=0}^{n} \in \mathbb{X}_{\mathrm{D}}^{+s}(\Sigma)$. In addition, note that $\mathbb{X}_{\mathrm{D}}^{+s}(\Sigma)$ and $\mathbb{X}_{\mathrm{N}}^{-s}(\Sigma)$ have only been defined for $s \in(0,1)$, see $(9)$ (10), however the previous observations suggest a natural extension of these 
definitions for $s \in(-1 / 2,0]$. Routine calculus allows to verify the following lemma.

\section{Lemma 3}

For any $s \in(-1 / 2,+1 / 2)$, the jump operator $\mathfrak{u}=\left(u_{j}\right)_{j=0}^{n} \mapsto\left(\left[u_{\mathrm{J}}\right]\right)_{\mathrm{J} \in \mathfrak{I}}$ continuously maps $\mathbb{H}^{s}(\Sigma)$ onto $\Pi_{\mathrm{J} \in \mathfrak{I}} \mathrm{H}^{s}\left(\Gamma_{\mathrm{J}}\right)$. Its kernel will be denoted $\mathbb{X}_{\mathrm{D}}^{s}(\Sigma)$, and it is a closed subspace of $\mathbb{H}^{s}(\Sigma)$.

Similarly, for $s \in(-1 / 2,+1 / 2)$, the operator $\mathfrak{u}=\left(u_{j}\right)_{j=0}^{n} \mapsto\left(\left\{u_{\mathrm{J}}\right\}\right)_{\mathrm{J} \in \mathfrak{I}}$ continuously maps $\mathbb{H}^{s}(\Sigma)$ onto $\Pi_{\mathrm{J} \in \mathfrak{I}} \mathrm{H}^{s}\left(\Gamma_{\mathrm{J}}\right)$. Its kernel will be denoted $\mathbb{X}_{\mathrm{N}}^{s}(\Sigma)$. For any $\mathfrak{u} \in \mathbb{H}^{s}(\Sigma)$ we have thus

$$
\begin{aligned}
& \mathfrak{u} \in \mathbb{X}_{\mathrm{D}}^{s}(\Sigma) \quad \Longleftrightarrow \quad\left[u_{\mathrm{J}}\right]=0 \quad \forall \mathrm{J} \in \mathfrak{I}, \\
& \mathfrak{u} \in \mathbb{X}_{\mathrm{N}}^{s}(\Sigma) \quad \Longleftrightarrow \quad\left\{u_{\mathrm{J}}\right\}=0 \quad \forall \mathrm{J} \in \mathfrak{I} .
\end{aligned}
$$

Let us emphasise that the definition of $\mathbb{X}_{\mathrm{D}}^{+s}(\Sigma)$ and $\mathbb{X}_{\mathrm{N}}^{-s}(\Sigma)$ provided by Lemma 3 is consistant with (9)-(10) for the case $s \in(0,1 / 2)$, and it extends these definitions to the case $s \in(-1 / 2,0]$. Straightforward algebraic calculus based on (25) yield the following result.

\section{Corollary 3}

For any $s \in(-1 / 2,+1 / 2)$ we have $\mathbb{H}^{s}(\Sigma)=\mathbb{X}_{\mathrm{D}}^{s}(\Sigma) \oplus \mathbb{X}_{\mathrm{N}}^{s}(\Sigma)$.

With the definitions provided by Lemma 3 , for $|s|<1 / 2$, the space $\mathbb{X}_{\mathrm{D}}^{-s}(\Sigma)$ can be considered as dual to $\mathbb{X}_{\mathrm{D}}^{+s}(\Sigma)$, and $\mathbb{X}_{\mathrm{N}}^{-s}(\Sigma)$ dual to $\mathbb{X}_{\mathrm{N}}^{+s}(\Sigma)$. Based on the previous corollary, it is natural to consider Formulation (19) with the choice $\mathbb{Y}^{s}(\Sigma)=\mathbb{X}_{\mathrm{N}}^{s}(\Sigma)$. Assuming that $\mathfrak{g}_{\mathrm{N}} \in \mathbb{X}_{\mathrm{N}}^{-s}(\Sigma)$ for some $s \in\left[s_{\star}, 1 / 2\right)$ where $s_{\star}$ is as in Theorem 1 (here in particular $s<1 / 2$ is assumed), it then simply writes as follows

$$
\left\{\begin{array}{l}
\text { Find } \mathfrak{p} \in \mathbb{X}_{\mathrm{N}}^{-s}(\Sigma) \text { such that } \\
\left\langle\left\langle\left(\operatorname{Id}-\mathbf{M}_{\mathrm{N}}\right) \cdot \mathbf{I}_{1 / \mu}(\mathfrak{p}), \mathfrak{v}\right\rangle\right\rangle=\left\langle\left\langle\mathfrak{g}_{\mathrm{N}}, \mathfrak{v}\right\rangle\right\rangle \quad \forall \mathfrak{v} \in \mathbb{X}_{\mathrm{N}}^{+s}(\Sigma)
\end{array}\right.
$$

In this formulation, what comes into play is the bilinear form $\mathfrak{u}, \mathfrak{v} \mapsto$ $\left\langle\left\langle\mathbf{I}_{1 / \mu}(\mathfrak{u}), \mathfrak{v}\right\rangle\right\rangle$ with $\mathfrak{u} \in \mathbb{X}_{\mathrm{N}}^{+s}(\Sigma), \mathfrak{v} \in \mathbb{X}_{\mathrm{N}}^{-s}(\Sigma)$ with $s \in\left[s_{\star}, 1 / 2\right)$, and not just $\langle\langle\mathfrak{u}, \mathfrak{v}\rangle\rangle$. Since $u_{\mathrm{J}_{ \pm}}= \pm\left[u_{\mathrm{J}}\right] / 2$ whenever $\mathfrak{u}=\left(u_{j}\right)_{j=0}^{n} \in \mathbb{X}_{\mathrm{N}}^{s}(\Sigma)$, this bilinear form decomposes as

$$
\begin{aligned}
\left\langle\left\langle\mathbf{I}_{1 / \mu}(\mathfrak{u}), \mathfrak{v}\right\rangle\right\rangle & =\sum_{j=0}^{n} \frac{1}{\mu_{j}}\left\langle u_{j}, v_{j}\right\rangle_{\Gamma_{j}}=\sum_{\mathrm{J} \in \mathfrak{I}} \frac{1}{\mu_{\mathrm{J}_{+}}}\left\langle u_{\mathrm{J}_{+}}, v_{\mathrm{J}_{+}}\right\rangle_{\Gamma_{\mathrm{J}}}+\frac{1}{\mu_{\mathrm{J}_{-}}}\left\langle u_{\mathrm{J}_{-}}, v_{\mathrm{J}_{-}}\right\rangle_{\Gamma_{\mathrm{J}}} \\
& =\sum_{\substack{\mathrm{J} \in \mathfrak{I} \\
\text { where }}} \frac{1}{2}\left\{\mu_{\mathrm{J}}^{-1}\right\}\left\langle\left[u_{\mathrm{J}}\right],\left[v_{\mathrm{J}}\right]\right\rangle_{\Gamma_{\mathrm{J}}} \quad \forall \mathfrak{u} \in \mathbb{X}_{\mathrm{N}}^{-s}(\Sigma), \forall \mathfrak{v} \in \mathbb{X}_{\mathrm{N}}^{+s}(\Sigma) \\
& =\left(\mu_{\mathrm{J}_{+}}^{-1}+\mu_{\mathrm{J}_{-}}^{-1}\right) / 2 .
\end{aligned}
$$


7.1 Decomposition of potentials

We can perform a similar decomposition on the multi-potential operator from (15). Indeed for $|s|<1 / 2$, and for any $\mathfrak{u}=\left(u_{j}\right)_{j=0}^{n} \in \mathbb{X}_{\mathrm{N}}^{s}(\Sigma)$, we have

$$
\sum_{j=0}^{n} \frac{1}{\mu_{j}} \mathrm{SL}_{j}\left(u_{j}\right)(\boldsymbol{x})=\sum_{\mathrm{J} \in \mathfrak{I}} \frac{1}{\mu_{\mathrm{J}_{+}}} \mathrm{SL}_{\mathrm{J}}\left(u_{\mathrm{J}_{+}}\right)(\boldsymbol{x})+\frac{1}{\mu_{\mathrm{J}_{-}}} \mathrm{SL}_{\mathrm{J}}\left(u_{\mathrm{J}_{-}}\right)(\boldsymbol{x})=\sum_{\mathrm{J} \in \mathfrak{I}} \frac{1}{2}\left[\mu_{\mathrm{J}}^{-1}\right] \mathrm{SL}_{\mathrm{J}}\left(\left[u_{\mathrm{J}}\right]\right)(\boldsymbol{x}),
$$

where $\left[\mu_{\mathrm{J}}^{-1}\right]:=\frac{1}{\mu_{\mathrm{J}_{+}}}-\frac{1}{\mu_{\mathrm{J}_{-}}} \quad$ and $\quad \mathrm{SL}_{\mathrm{J}}(p)(\boldsymbol{x}):=\int_{\Gamma_{\mathrm{J}}} \frac{p(\boldsymbol{y}) d \sigma(\boldsymbol{y})}{4 \pi|\boldsymbol{x}-\boldsymbol{y}|}$.

Considering $\mathrm{H}^{s}\left(\Gamma_{\mathrm{J}}\right)$ as a subspace of $\mathrm{H}^{s}\left(\Gamma_{\mathrm{J}_{+}}\right)$or $\mathrm{H}^{s}\left(\Gamma_{\mathrm{J}_{-}}\right)$, the defintion of $\mathrm{SL}_{\mathrm{J}}$ makes sense according to (23). Moreover it continuously maps $\mathrm{H}^{s}\left(\Gamma_{\mathrm{J}}\right)$ into $\mathrm{H}^{s+3 / 2}\left(\Delta, \Omega_{\mathrm{J}_{ \pm}}\right)$according to $[16$, Thm.1]. Next fix an arbitrary Q $\in \mathfrak{I}$ and observe that, for any $\mathrm{J} \in \mathfrak{I}$, we have $\left(\gamma_{\mathrm{N}}^{\mathrm{J}_{+}}+\gamma_{\mathrm{N}}^{\mathrm{J}_{-}}\right) \cdot \mathrm{SL}_{\mathrm{Q}}(p)=0$ on $\Gamma_{\mathrm{J}}$ if $\mathrm{J} \neq \mathrm{Q}$, and $\left(\gamma_{\mathrm{N}}^{\mathrm{Q}_{+}}+\gamma_{\mathrm{N}}^{\mathrm{Q}_{-}}\right) \cdot \mathrm{SL}_{\mathrm{Q}}(p)=p$ on $\Gamma_{\mathrm{Q}}$, for all $p \in \mathrm{H}^{s}\left(\Gamma_{\mathrm{Q}}\right)$. As a consequence, taking account of (28) for the expression of $\mathbf{M}_{\mathrm{N}}$, for any $|s|<1 / 2$, any $\mathfrak{u}=\left(u_{j}\right)_{j=0}^{n} \in \mathbb{X}_{\mathrm{N}}^{-s}(\Sigma)$ and any $\mathfrak{v}=\left(v_{j}\right)_{j=0}^{n} \in \mathbb{X}_{\mathrm{N}}^{+s}(\Sigma)$ we have

$$
\begin{aligned}
\left\langle\left\langle\mathbf{M}_{\mathrm{N}} \cdot \mathbf{I}_{1 / \mu}(\mathfrak{u}), \mathfrak{v}\right\rangle\right\rangle & =\sum_{\mathrm{Q} \in \mathfrak{I}} \frac{1}{2}\left[\mu_{\mathrm{Q}}^{-1}\right] \sum_{j=0}^{n}\left\langle\gamma_{\mathrm{N}}^{j} \cdot \mathrm{SL}_{\mathrm{Q}}\left(\left[u_{\mathrm{Q}}\right]\right), v_{j}\right\rangle_{\Gamma_{j}} \\
& =\sum_{\mathrm{Q} \in \mathfrak{I}} \frac{1}{2}\left[\mu_{\mathrm{Q}}^{-1}\right] \sum_{\mathrm{J} \in \mathfrak{I}}\left\langle\gamma_{\mathrm{N}}^{\mathrm{J}_{+}} \cdot \mathrm{SL}_{\mathrm{Q}}\left(\left[u_{\mathrm{Q}}\right]\right), v_{\mathrm{J}_{+}}\right\rangle_{\Gamma_{\mathrm{J}}}+\left\langle\gamma_{\mathrm{N}}^{\mathrm{J}_{-}} \cdot \mathrm{SL}_{\mathrm{Q}}\left(\left[u_{\mathrm{Q}}\right]\right), v_{\mathrm{J}_{-}}\right\rangle_{\Gamma_{\mathrm{J}}} \\
& =\sum_{\mathrm{Q} \in \mathfrak{I}} \sum_{\mathrm{J} \in \mathfrak{I}} \frac{1}{2}\left[\mu_{\mathrm{Q}}^{-1}\right]\left\langle\left\{\gamma_{\mathrm{N}}^{\mathrm{J}}\right\} \cdot \mathrm{SL}_{\mathrm{Q}}\left(\left[u_{\mathrm{Q}}\right]\right),\left[v_{\mathrm{J}}\right]\right\rangle_{\Gamma_{\mathrm{J}}}
\end{aligned}
$$

where we have used the notations $\left\{\gamma_{\mathrm{N}}^{\mathrm{J}}\right\} \cdot \psi:=\left(\gamma_{\mathrm{N}}^{\mathrm{J}_{+}}(\psi)-\gamma_{\mathrm{N}}^{\mathrm{J}_{-}}(\psi)\right) / 2$ and $\left[\mu_{\mathrm{Q}}^{-1}\right]:=$ $\mu_{\mathrm{Q}_{+}}^{-1}-\mu_{\mathrm{Q}_{-}}^{-1}$. The potential operators $\left\{\gamma_{\mathrm{N}}^{\mathrm{J}}\right\} \cdot \mathrm{SL}_{\mathrm{Q}}$ admit a very explicit expression as a Cauchy principal value integral

$$
\left\{\gamma_{\mathrm{N}}^{\mathrm{J}}\right\} \cdot \mathrm{SL}_{\mathrm{Q}}(p)(\boldsymbol{x}):=\lim _{\epsilon \rightarrow 0} \int_{\Gamma_{\mathrm{Q}} \backslash \mathrm{B}_{\epsilon}(\boldsymbol{x})} \frac{\mathbf{n}_{\mathrm{J}_{+}}(\boldsymbol{x}) \cdot(\boldsymbol{y}-\boldsymbol{x})}{4 \pi|\boldsymbol{y}-\boldsymbol{x}|^{3}} p_{\mathrm{Q}}(\boldsymbol{y}) d \sigma(\boldsymbol{y}) \quad \forall \boldsymbol{x} \in \Gamma_{\mathrm{J}}
$$

where $\mathbf{n}_{\mathrm{J}_{+}}$refers to the normal vector to $\Omega_{\mathrm{J}_{+}}$directed toward the exterior of $\Omega_{\mathrm{J}_{+}}$. Combining (27) with (28) and (29), we finally obtain: for $|s|<1 / 2$ and for all $\mathfrak{u} \in \mathbb{X}_{\mathrm{N}}^{-s}(\Sigma), \mathfrak{v} \in \mathbb{X}_{\mathrm{N}}^{+s}(\Sigma)$, we have

$$
\begin{aligned}
\left\langle\left\langle\left(\mathrm{Id}-\mathbf{M}_{\mathrm{N}}\right) \cdot \mathbf{I}_{1 / \mu}(\mathfrak{u}), \mathfrak{v}\right\rangle\right\rangle= & \sum_{\mathrm{J} \in \mathfrak{I}} \frac{1}{2}\left\{\mu_{\mathrm{J}}^{-1}\right\}\left\langle\left[u_{\mathrm{J}}\right],\left[v_{\mathrm{J}}\right]\right\rangle_{\Gamma_{J}}-\sum_{\mathrm{Q} \in \mathfrak{I}} \sum_{\mathrm{J} \in \mathfrak{I}} \frac{1}{2}\left[\mu_{\mathrm{Q}}^{-1}\right]\left\langle\left\{\gamma_{\mathrm{N}}^{\mathrm{J}}\right\} \cdot \mathrm{SL}_{\mathrm{Q}}\left(\left[u_{\mathrm{Q}}\right]\right),\left[v_{\mathrm{J}}\right]\right\rangle_{\Gamma_{\mathrm{J}}} \\
= & \sum_{\mathrm{J} \in \mathfrak{I}}\left\{\mu_{\mathrm{J}}^{-1}\right\}\left\langle p_{\mathrm{J}}, q_{\mathrm{J}}\right\rangle_{\Gamma_{J}}-\sum_{\mathrm{Q} \in \mathfrak{I}} \sum_{\mathrm{J} \in \mathfrak{I}}\left[\mu_{\mathrm{Q}}^{-1}\right]\left\langle\left\{\gamma_{\mathrm{N}}^{\mathrm{J}}\right\} \cdot \mathrm{SL}_{\mathrm{Q}}\left(p_{\mathrm{Q}}\right), q_{\mathrm{J}}\right\rangle_{\Gamma_{\mathrm{J}}} \\
& \text { with } \quad p_{\mathrm{J}}:=\left[u_{\mathrm{J}}\right] \quad \text { and } \quad q_{\mathrm{J}}:=\left[v_{\mathrm{J}}\right] / 2 .
\end{aligned}
$$




\subsection{Final reformulation}

Analogous calculus can be achieved for reducing the right hand side in (26), taking account that $\mathfrak{g}_{\mathrm{N}} \in \mathbb{X}_{\mathrm{N}}^{-s}(\Sigma)$ for some $s \in\left[s_{\star}, 1 / 2\right)$. Formulation (26) is then ultimately reduced to the following

$$
\begin{aligned}
& \text { Find } \mathfrak{p}=\left(p_{\mathrm{J}}\right) \in \Pi_{\mathrm{J} \in \mathfrak{I}} \mathrm{H}^{-s}\left(\Gamma_{\mathrm{J}}\right) \text { such that } \\
& \sum_{\mathrm{J} \in \mathfrak{I}}\left\{\mu_{\mathrm{J}}^{-1}\right\}\left\langle p_{\mathrm{J}}, q_{\mathrm{J}}\right\rangle_{\Gamma_{J}}-\sum_{\mathrm{Q} \in \mathfrak{I}} \sum_{\mathrm{J} \in \mathfrak{I}}\left[\mu_{\mathrm{Q}}^{-1}\right]\left\langle\left\{\gamma_{\mathrm{N}}^{\mathrm{J}}\right\} \cdot \mathrm{SL}_{\mathrm{Q}}\left(p_{\mathrm{Q}}\right), q_{\mathrm{J}}\right\rangle_{\Gamma_{\mathrm{J}}}=\sum_{\mathrm{J} \in \mathfrak{I}}\left\langle\left[g_{\mathrm{J}}\right], q_{\mathrm{J}}\right\rangle_{\Gamma_{\mathrm{J}}} \\
& \forall \mathfrak{q}=\left(q_{\mathrm{J}}\right) \in \Pi_{\mathrm{J} \in \mathfrak{I}} \mathrm{H}^{+s}\left(\Gamma_{\mathrm{J}}\right)
\end{aligned}
$$

In accordance with $(28)$, if $\mathfrak{p}=\left(p_{\mathrm{J}}\right)_{\mathrm{J} \in \mathfrak{I}}$ is solution to Formulation (31), then the function

$$
u(\boldsymbol{x})=\frac{1}{2} \sum_{\mathrm{J} \in \mathfrak{I}}\left[\mu_{\mathrm{J}}^{-1}\right] \mathrm{SL}_{\mathrm{J}}\left(p_{\mathrm{J}}\right)(\boldsymbol{x}), \quad \boldsymbol{x} \in \mathbb{R}^{3} \backslash \Sigma
$$

is the solution of Problem (1). Of course, since we only transformed (26) by means of elementary algebraic manipulations, (31) admits a unique solution, and the operator associated to the bilinear form in the left hand side isomorphically maps $\Pi_{\mathrm{J} \in \mathfrak{I}} \mathrm{H}^{-s}\left(\Gamma_{\mathrm{J}}\right)$ into $\Pi_{\mathrm{J} \in \mathfrak{I}} \mathrm{H}^{-s}\left(\Gamma_{\mathrm{J}}\right)$.

Remark 2 In Formulation (31) the solution for $\mathfrak{p}$ provides the normal normal flux of the solution $u$ of the transmission problem (3) on interfaces. Thus, (31) qualifies as a direct boundary integral equation. Dirichlet traces of $u$ on interfaces have to be recovered through (32).

\section{Formulation in square integrable function spaces}

The space of square integrable functions is a more natural and convenient functional setting when considering boundary integral equations of the second kind. It is indeed a well established result, see $[15,46,14]$, that the Dirichlet trace of the double layer potential continuously maps square integrable traces to square integrable traces. The next proposition is a direct application of [16, Thm.1].

\section{Proposition 6}

For any $j=0 \ldots n$, the operators $\gamma_{\mathrm{N}}^{j} \cdot \mathrm{SL}_{j}$ and $\gamma_{\mathrm{D}}^{j} \cdot \mathrm{DL}_{j}$ continuously map $\mathrm{L}^{2}\left(\Gamma_{j}\right)$ into $\mathrm{L}^{2}\left(\Gamma_{j}\right)$.

We wish to show that (19) can be reformulated choosing square integrable trial and test functions. In the present context, we need to consider maps of the form $\gamma_{\mathrm{N}}^{k} \cdot \mathrm{SL}_{j}$ for $k=j$, but also for $k \neq j$. Hence a natural question is wether such a continuity result as Proposition 6 holds also for $k \neq j$. This clearly holds whenever $\bar{\Gamma}_{j} \cap \bar{\Gamma}_{k}=\emptyset$ due to the regularity of the Green kernel 
$\mathscr{G}(\boldsymbol{x})=1 /(4 \pi|\boldsymbol{x}|)$ for $\boldsymbol{x} \neq 0$. Conversely, it is not obvious, if $\bar{\Gamma}_{j} \cap \bar{\Gamma}_{k} \neq \emptyset$, even if $\Gamma_{j}$ and $\Gamma_{k}$ have only an edge in common.

To study this problem we resort on a result of Dahlberg [19, Thm.1] concerning harmonic measures. We first recall the definition of such measures. If $\mathcal{O} \subset \mathbb{R}^{3}$ is any bounded Lipschitz open set, for any $f \in \mathscr{C}^{0}(\partial \mathcal{O})$ let $\mathcal{P}(\mathcal{O}, f) \in \mathrm{L}^{2}(\mathcal{O})$ refer to the unique function satisfying

$$
\Delta \mathcal{P}(\mathcal{O}, f)=0 \quad \text { in } \mathcal{O} \quad \text { and } \quad \mathcal{P}(\mathcal{O}, f)=f \quad \text { on } \partial \mathcal{O} .
$$

For any $\boldsymbol{x} \in \mathcal{O}$ the mapping $f \mapsto \mathcal{P}(\mathcal{O}, f)(\boldsymbol{x})$ is a continuous functional on $\mathscr{C}^{0}(\partial \mathcal{O})$ which, due to Riesz representation theorem [41, Thm.6.19], is associated to the so-called harmonic measure $d \omega(\mathcal{O}, \boldsymbol{x})$ on $\partial \mathcal{O}$ via the formula

$$
\mathcal{P}(\mathcal{O}, f)(\boldsymbol{x})=\int_{\partial \mathcal{O}} f d \omega(\mathcal{O}, \boldsymbol{x}) \quad \forall \boldsymbol{x} \in \mathcal{O} .
$$

Precise description of harmonic measures associated to Lipschitz domains in terms of Green functions were provided in [18, Thm.3]. The result below, established in [19, Thm.1], bounds harmonic measures inside its domain of definition. We do not formulate this theorem in full generality, but restate it so as to fit our present problem.

\section{Theorem 2}

Let $\Omega \subset \mathbb{R}^{3}$ be a bounded Lipschitz domain. Let $m$ refer to any positive measure on $\Omega$, such that $\lim \sup _{r \rightarrow 0} r^{-2} m\left(\mathrm{~B}_{r}(\boldsymbol{x}) \cap \Omega\right)<+\infty$ for all $\boldsymbol{x} \in \partial \Omega$. Then there exists a constant $C>0$ such that

$$
\int_{\Omega}|\mathcal{P}(\Omega, f)|^{2} d m \leq C\|f\|_{\mathrm{L}^{2}(\partial \Omega)}^{2} \quad \forall f \in \mathrm{L}^{2}(\partial \Omega) .
$$

In this theorem $\mathrm{B}_{r}(\boldsymbol{x})$ is the ball of radius $r$ centred at $\boldsymbol{x}$, and $\mathrm{L}^{2}(\partial \Omega)$ refers to the classical space of (almost everywhere defined) square integrable functions with respect to the surface Lebesgue measure on $\partial \Omega$. In Theorem 2 the measure $m$ does not necessarily refer to the classical Lebesgue measure on $\mathbb{R}^{3}$ that actually satisfies the stronger estimate $\limsup _{r \rightarrow 0} r^{-3} m\left(\mathrm{~B}_{r}(\boldsymbol{x}) \cap \Omega\right)<+\infty$. Here, we are more interested in the case where $m$ is related to the surface measure of the subdomains $\Omega_{j}$.

\section{Proposition 7}

The operator $\gamma_{\mathrm{D}}^{j} \cdot \mathrm{DL}_{k}$ continuously maps $\mathrm{L}^{2}\left(\Gamma_{k}\right)$ into $\mathrm{L}^{2}\left(\Gamma_{j}\right) \forall j, k=0 \ldots n$.

\section{Proof:}

The case $j=k$ is already covered by Proposition 6 . On the other hand, the case where $\bar{\Gamma}_{j} \cap \bar{\Gamma}_{k}=\emptyset$ is trivial. So we only need to concentrate on the case where $\bar{\Gamma}_{j} \cap \bar{\Gamma}_{k} \neq \emptyset$ and $\Omega_{j} \neq \Omega_{k}$.

Let B refer to an open ball such that $\cup_{j=1}^{n} \bar{\Omega}_{j} \subset \mathrm{B}$. Denote $\sigma_{j}$ the surface Lebesgue measure on $\Gamma_{j}$ and let $m_{j}$ refer to the unique Borel measure on 
$\mathbb{R}^{3}$ satisfying $m_{j}(U):=\sigma_{j}\left(\Gamma_{j} \cap U\right)$ for all open sets $U \subset \mathbb{R}^{3}$. Fix $j, k$ arbitrarily, and set $\Omega_{j}^{\prime}=\mathrm{B} \backslash \bar{\Omega}_{j}$ and $\Gamma_{j}^{\prime}=\Gamma_{j} \cup \partial \mathrm{B}=\partial \Omega_{j}^{\prime}$. Since obviously $\limsup _{r \rightarrow 0} r^{-2} m_{k}\left(\mathrm{~B}_{r}(\boldsymbol{x}) \cap \Omega_{j}^{\prime}\right) \leq \limsup _{r \rightarrow 0} r^{-2} m_{k}\left(\mathrm{~B}_{r}(\boldsymbol{x})\right)<+\infty$, we can apply Theorem 2 with the choice $\Omega=\Omega_{j}^{\prime}$ and $m=m_{k}$, which yields

$$
\int_{\Gamma_{k} \backslash \Gamma_{j}}\left|\mathcal{P}\left(\Omega_{j}^{\prime}, f\right)\right|^{2} d \sigma_{k} \leq C_{j, k}\|f\|_{L^{2}\left(\Gamma_{j}^{\prime}\right)}^{2} \quad \forall f \in \mathrm{L}^{2}\left(\Gamma_{j}^{\prime}\right) .
$$

This estimate shows in particular that, if $u \in \mathrm{H}^{1}\left(\Omega_{j}^{\prime}\right)$ satisfies $\Delta u=0$ in $\Omega_{j}^{\prime}$, then $f=\left.u\right|_{\Gamma_{j}^{\prime}} \in \mathrm{H}^{1 / 2}\left(\Gamma_{j}^{\prime}\right) \subset \mathrm{L}^{2}\left(\Gamma_{j}^{\prime}\right)$ and we have $\mathcal{P}\left(\left.u\right|_{\Gamma_{j}^{\prime}}, \Omega_{j}^{\prime}\right)=u$ in $\Omega_{j}^{\prime}$. This leads to

$$
\begin{aligned}
& \|u\|_{\mathrm{L}^{2}\left(\Gamma_{k}\right)}^{2} \leq\left(1+C_{j, k}\right)\|u\|_{\mathrm{L}^{2}\left(\Gamma_{j}^{\prime}\right)}^{2} \\
& \forall u \in \mathrm{H}^{1}\left(\Gamma_{j}^{\prime}\right) \text { satisfying } \Delta u=0 \text { in } \Omega_{j}^{\prime} .
\end{aligned}
$$

Now consider the particular choice $u=\mathrm{DL}_{j}(p)$ for some $p \in \mathrm{H}^{1 / 2}\left(\Gamma_{j}\right)$. Clearly $\left\|\mathrm{DL}_{j}(p)\right\|_{\mathrm{L}^{2}(\partial \mathrm{B})} \leq C_{j}\|p\|_{\mathrm{L}^{2}\left(\Gamma_{j}\right)} \forall p \in \mathrm{H}^{1 / 2}\left(\Gamma_{j}\right)$ for some fixed constant $C_{j}>$ 0 that only depends on $j$, due to the regularity of the Green kernel, since $\Gamma_{j} \cap \partial \mathrm{B}=\emptyset$. This finally leads to the existence of a constant $C>0$ such that $\left\|\gamma_{\mathrm{D}}^{k} \cdot \mathrm{DL}_{j}(p)\right\|_{\mathrm{L}^{2}\left(\Gamma_{k}\right)} \leq C\|p\|_{\mathrm{L}^{2}\left(\Gamma_{j}\right)} \quad \forall p \in \mathrm{L}^{2}\left(\Gamma_{j}\right)$.

It is important to note that the previous proposition holds even if $\Gamma_{j} \cap \Gamma_{k} \neq \emptyset$ and $\Gamma_{j} \neq \Gamma_{k}$. A comparable continuity result also holds for the single layer potential.

\section{Corollary 4}

The operator $\gamma_{\mathrm{N}}^{j} \cdot \mathrm{SL}_{k}$ continuously maps $\mathrm{L}^{2}\left(\Gamma_{k}\right)$ into $\mathrm{L}^{2}\left(\Gamma_{j}\right) \forall j, k=0 \ldots n$

\section{Proof:}

We will rely on the formal adjointness of $\gamma_{\mathrm{N}}^{j} \cdot \mathrm{SL}_{k}$ with $-\gamma_{\mathrm{D}}^{k} \cdot \mathrm{DL}_{j}$. Let $\mathcal{O}$ be an open set such that $\Gamma_{j} \cap \Gamma_{k} \subset \mathcal{O}$. Consider two functions $u \in \mathrm{L}^{2}\left(\Gamma_{j}\right)$ and $v \in \mathrm{L}^{2}\left(\Gamma_{k}\right)$, such that $u=0$ on $\Gamma_{j} \cap \mathcal{O}$. Due to the regularity of the Green kernel we have

$$
\begin{aligned}
\left\langle\gamma_{\mathrm{N}}^{j} \cdot \mathrm{SL}_{k}(v), u\right\rangle_{\Gamma_{j}} & =\int_{\Gamma_{j} \backslash \Gamma_{k}}\left(\int_{\Gamma_{k}} \frac{\mathbf{n}_{j}(\boldsymbol{x}) \cdot(\boldsymbol{y}-\boldsymbol{x})}{4 \pi|\boldsymbol{y}-\boldsymbol{x}|^{3}} v(\boldsymbol{y}) d \sigma_{k}(\boldsymbol{y})\right) u(\boldsymbol{x}) d \sigma_{j}(\boldsymbol{x}) \\
& =-\int_{\Gamma_{k}}\left(\int_{\Gamma_{j} \backslash \Gamma_{k}} \frac{\mathbf{n}_{j}(\boldsymbol{x}) \cdot(\boldsymbol{x}-\boldsymbol{y})}{4 \pi|\boldsymbol{y}-\boldsymbol{x}|^{3}} u(\boldsymbol{x}) d \sigma_{j}(\boldsymbol{x})\right) v(\boldsymbol{y}) d \sigma_{k}(\boldsymbol{y}) \\
& =-\left\langle v, \gamma_{\mathrm{D}}^{k} \cdot \mathrm{DL}_{j}(u)\right\rangle_{\Gamma_{k}}
\end{aligned}
$$

From this, together with Proposition 7, we obtain the existence of a constant $C>0$ not depending on $\mathcal{O}$ such that $\left|\left\langle\gamma_{\mathrm{N}}^{j} \cdot \mathrm{SL}_{k}(v), u\right\rangle_{\Gamma_{j}}\right| \leq C\|u\|_{\mathrm{L}^{2}\left(\Gamma_{j}\right)}\|v\|_{\mathrm{L}^{2}\left(\Gamma_{k}\right)}$. Since the constant $C$ does not depend on $\mathcal{O}$, using dominated convergence theorem, we conclude that

$$
\left|\left\langle\gamma_{\mathrm{N}}^{j} \cdot \mathrm{SL}_{k}(v), u 1_{\Gamma_{j} \backslash \Gamma_{k}}\right\rangle_{\Gamma_{j}}\right| \leq C\|u\|_{\mathrm{L}^{2}\left(\Gamma_{j}\right)}\|v\|_{\mathrm{L}^{2}\left(\Gamma_{k}\right)} \quad \forall u \in \mathrm{L}^{2}\left(\Gamma_{j}\right), \forall v \in \mathrm{L}^{2}\left(\Gamma_{k}\right) .
$$


Next on $\Gamma_{j} \cap \Gamma_{k}$, we have $\gamma_{\mathrm{N}}^{j}=-\gamma_{\mathrm{N}, c}^{k}$. Since $\gamma_{\mathrm{N}, c}^{k} \cdot \mathrm{SL}_{k}=-\mathrm{Id}+\gamma_{\mathrm{N}}^{k} \cdot \mathrm{SL}_{k}$ continuously maps $\mathrm{L}^{2}\left(\Gamma_{k}\right)$ into $\mathrm{L}^{2}\left(\Gamma_{k}\right)$ according to Proposition 6 , we conclude that $1_{\Gamma_{j} \cap \Gamma_{k}} \gamma_{\mathrm{N}}^{j} \cdot \mathrm{SL}_{k}$ continuously maps $\mathrm{L}^{2}\left(\Gamma_{k}\right)$ into $\mathrm{L}^{2}\left(\Gamma_{j}\right)$. To finish the proof, observe that any function $u \in \mathrm{L}^{2}\left(\Gamma_{j}\right)$ can be decomposed as $u=u 1_{\Gamma_{j} \backslash \Gamma_{k}}+$ $u 1_{\Gamma_{j} \cap \Gamma_{k}}$.

The continuity results established above suggest that we consider Formulation (31) in the framework of square integrable traces. Define

$$
\begin{aligned}
& \mathbb{L}^{2}(\Sigma):=\mathrm{L}^{2}\left(\Gamma_{0}\right) \times \cdots \times \mathrm{L}^{2}\left(\Gamma_{n}\right) \\
& \text { with } \quad\|\mathfrak{v}\|_{\mathbb{L}^{2}(\Sigma)}^{2}=\left\|v_{0}\right\|_{\mathrm{L}^{2}\left(\Gamma_{0}\right)}^{2}+\cdots+\left\|v_{n}\right\|_{\mathrm{L}^{2}\left(\Gamma_{n}\right)}^{2} \\
& \text { and } \quad \mathrm{L}^{2}(\Sigma):=\left\{\mathfrak{v}=\left(v_{j}\right)_{j=0}^{n} \in \mathbb{L}^{2}(\Sigma) \mid v_{j}=v_{k} \text { on } \Gamma_{j} \cap \Gamma_{k} \forall j, k\right\}
\end{aligned}
$$

The set $\mathbb{L}^{2}(\Sigma)$ is the space $\mathbb{H}^{s}(\Sigma)$ for $s=0$. As such, it is equipped with the pairing $\left\langle\langle\right.$,$\rangle , and (\mathfrak{u}, \mathfrak{v})_{\mathbb{L}^{2}(\Sigma)}=\langle\langle\mathfrak{u}, \overline{\mathfrak{v}}\rangle\rangle$ is the scalar product associated with the norm \|\|$_{\mathbb{L}^{2}(\Sigma)}$. Moreover $\mathrm{L}^{2}(\Sigma) \subset \mathbb{L}^{2}(\Sigma)$ is a closed subspace. The singletrace space admits a natural counterpart in this new setting. Observe indeed that

$$
\begin{array}{ll}
\mathrm{L}^{2}(\Sigma)=\mathbb{L}^{2}(\Sigma) \cap \mathbb{X}_{\mathrm{D}}^{-s}(\Sigma) & \text { and } \\
\mathrm{L}^{2}(\Sigma)^{\perp}=\mathbb{L}^{2}(\Sigma) \cap \mathbb{X}_{\mathrm{N}}^{-s}(\Sigma) & \forall s \in[0,1 / 2)
\end{array}
$$

where $\mathrm{L}^{2}(\Sigma)^{\perp}$ refers to the space orthogonal to $\mathrm{L}^{2}(\Sigma)$ with respect to the scalar product $(,)_{\mathbb{L}^{2}(\Sigma)}$. As regards the multi-potential operator involved in the boundary integral formulation (16), we have a continuity result as a direct application of Corollary 4. This operator also satisfies Corollary 2 and Proposition 4 in this new setting.

\section{Proposition 8}

The operator $\mathbf{M}_{\mathrm{N}}$ continuously maps $\mathbb{L}^{2}(\Sigma)$ into $\mathbb{L}^{2}(\Sigma)$. We have $\left\langle\left\langle\left(\operatorname{Id}-\mathbf{M}_{\mathrm{N}}\right) \mathfrak{p}, \mathfrak{v}\right\rangle=\right.$ $0 \forall \mathfrak{p} \in \mathbb{L}^{2}(\Sigma), \forall \mathfrak{v} \in \mathrm{L}^{2}(\Sigma)$. Moreover for any $\mathfrak{p} \in \mathbb{L}^{2}(\Sigma)$ we have $\mathbf{M}_{\mathrm{N}}(\mathfrak{p})=0$, if and only if $\mathfrak{p} \in \mathrm{L}^{2}(\Sigma)^{\perp}$.

Since $\mathbb{X}_{\mathrm{D}}^{s}(\Sigma)$ is dense in $\mathrm{L}^{2}(\Sigma)$, the first part of the proof is obtained directly by combining Proposition 4 with this density result. The second part results from algebraic manipulations like for Corollary 2.

\section{Proposition 9}

Assume that the solution $u \in \mathrm{H}_{\mathrm{loc}}^{1}\left(\mathbb{R}^{3}\right)$ to Problem (1) satisfies $\gamma_{\mathrm{N}}^{j}(u) \in \mathrm{L}^{2}\left(\Gamma_{j}\right)$ for all $j=0 \ldots n$. Then the tuple $\mathfrak{p}=\left(p_{j}\right)_{j=0}^{n} \in \mathbb{L}^{2}(\Sigma)$ defined by $p_{j}=\mu_{j} \gamma_{\mathrm{N}}^{j}(u)$ solves

$$
\mathfrak{p} \in \mathrm{L}^{2}(\Sigma)^{\perp} \quad \text { and } \quad\left\langle\left\langle\left(\mathrm{Id}-\mathbf{M}_{\mathrm{N}}\right) \mathbf{I}_{1 / \mu}(\mathfrak{p}), \mathfrak{v}\right\rangle\right\rangle=\left\langle\left\langle\mathfrak{g}_{\mathrm{N}}, \mathfrak{v}\right\rangle\right\rangle \quad \forall \mathfrak{v} \in \mathrm{L}^{2}(\Sigma)^{\perp}
$$

Proof:

Since $u_{\infty} \in \mathrm{H}_{\text {loc }}^{2}\left(\mathbb{R}^{3}\right)$, we have $g_{j}:=\gamma_{\mathrm{N}}^{j}\left(u_{\infty}\right) \in \mathrm{L}^{2}\left(\Gamma_{j}\right)$. Hence $\mathfrak{g}_{\mathrm{N}}=$ $\left(\mathfrak{g}_{j}\right)_{j=0}^{n} \in \mathrm{L}^{2}(\Sigma)^{\perp}=\mathbb{L}^{2}(\Sigma) \cap \mathbb{X}^{-1 / 2}(\Sigma)$. Also we know that $\mathfrak{p} \in \mathbb{X}_{\mathrm{N}}^{-1 / 2}(\Sigma)$ since 
$\mathbb{L}^{2}(\Sigma)^{\perp} \subset \mathbb{X}_{\mathrm{N}}^{-1 / 2}(\Sigma)$. Thus, according to $(16)$, it satisfies $\left\langle\left\langle\left(\operatorname{Id}-\mathbf{M}_{\mathrm{N}}\right) \mathbf{I}_{1 / \mu}(\mathfrak{p}), \mathfrak{v}\right\rangle\right\rangle=$ $\left\langle\left\langle\mathfrak{g}_{\mathrm{N}}, \mathfrak{v}\right\rangle\right\rangle \forall \mathfrak{v} \in \mathbb{H}^{1 / 2}(\Sigma)$. Then since $\mathfrak{g}_{\mathrm{N}} \in \mathbb{L}^{2}(\Sigma)$, and $\left(\operatorname{Id}-\mathbf{M}_{\mathrm{N}}\right) \mathbf{I}_{1 / \mu}(\mathfrak{p}) \in \mathbb{L}^{2}(\Sigma)$ according to Proposition 8 , and since $\mathbb{H}^{1 / 2}(\Sigma)$ is dense in $\mathbb{L}^{2}(\Sigma)$ for the norm \|\|$_{\mathbb{L}^{2}(\Sigma)}$, we conclude that

$$
\left\langle\left\langle\left(\operatorname{Id}-\mathbf{M}_{\mathrm{N}}\right) \mathbf{I}_{1 / \mu}(\mathfrak{p}), \mathfrak{v}\right\rangle\right\rangle=\left\langle\left\langle\mathfrak{g}_{\mathrm{N}}, \mathfrak{v}\right\rangle\right\rangle \quad \forall \mathfrak{v} \in \mathbb{L}^{2}(\Sigma)
$$

Next $\left(\operatorname{Id}-\mathbf{M}_{\mathrm{N}}\right) \mathbf{I}_{1 / \mu}(\mathfrak{p}) \in \mathrm{L}^{2}(\Sigma)^{\perp}$ according to Proposition 8 , and $\mathfrak{g}_{\mathrm{N}} \in \mathrm{L}^{2}(\Sigma)^{\perp}$. As a consequence (34) yields the trivial equation " $0=0$ " when choosing $\mathfrak{v} \in \mathrm{L}^{2}(\Sigma)$. So it is sufficient to consider $\mathfrak{v} \in \mathrm{L}^{2}(\Sigma)^{\perp}$.

\section{Galerkin discretisation}

We confine ourselves to subdomains that are curvilinear Lipschitz polyhedra, which covers most shapes occurring in engineering designs. Galerkin boundary element discretisation of (31) is based on a mesh partition of the skeleton $\Sigma$ that resolves the interfaces in the following sense: each interface $\Gamma_{\mathrm{J}}, \mathrm{J} \in \mathfrak{I}$, is partitioned into curvilinear polygons $\tau$, called elements, such that $\bar{\Gamma}_{\mathrm{J}}=$ $\cup_{\tau \in \mathcal{T}\left(\Gamma_{\mathrm{J}}\right)} \bar{\tau}$, where $\mathcal{T}\left(\Gamma_{\mathrm{J}}\right)$ is the "interface mesh", that is, the set of all elements paving $\Gamma_{\mathrm{J}}$. Then the skeleton mesh $\mathcal{T}(\Sigma)$ is the union of all these interface meshes. The interface meshes can be fairly arbitrary. In particular, "hanging nodes" are not excluded.

As finite-dimensional subspaces $\mathrm{H}_{\mathrm{N}}^{h}\left(\Gamma_{\mathrm{J}}\right)$ of $\mathrm{H}^{-s}\left(\Gamma_{\mathrm{J}}\right)$ and $\mathrm{H}^{+s}\left(\Gamma_{\mathrm{J}}\right)$ alike we choose spaces of discontinuous piecewise polynomials on the mesh $\mathcal{T}\left(\Gamma_{\mathrm{J}}\right)$. The degree of these polynomials can vary between different elements. Taking the product of all these interface boundary element spaces yields the final trial and test space $\mathrm{H}_{\mathrm{N}}^{h}(\Sigma)$.

Proposition 5 asserts existence and uniqueness of solutions of the second kind boundary integral equation (31), but for want of compactness of the operator $\mathbf{M}_{\mathrm{N}}: \mathbb{X}_{\mathrm{N}}^{-s}(\Sigma) \rightarrow \mathbb{X}_{\mathrm{N}}^{-s}(\Sigma)$ this does not imply well-posedness of the discrete variational problem, regardless of the resolution of the boundary element spaces: the numerical analysis of the discretised BIE remains an open problem. Yet, strong empirical evidence given in Section 10 bolsters our conjecture that Galerkin boundary element discretisation is uniformly stable in $\mathbb{L}^{2}(\Sigma)$ :

Conjecture 1 Let $B(\cdot, \cdot)$ stand for bilinear form of the variational BIE (31). Then we assume that

$$
\sup _{\mathfrak{q}_{h} \in \mathrm{H}_{\mathrm{N}}^{h}(\Sigma)} \frac{B\left(\mathfrak{p}_{h}, \mathfrak{q}_{h}\right)}{\left\|\mathfrak{q}_{h}\right\|_{\mathbb{L}^{2}(\Sigma)}} \geq c\left\|\mathfrak{p}_{h}\right\|_{\mathbb{L}^{2}(\Sigma)} \quad \forall \mathfrak{p}_{h} \in \mathrm{H}_{\mathrm{N}}^{h}(\Sigma),
$$

with $c>0$ independent of discretisation parameters like meshwidth and (local) polynomial degree. 
Firstly, if we take this assumption for granted, then, thanks to the $\mathbb{L}^{2}(\Sigma)$ continuity result of Proposition 8, we can conclude quasi-optimality of Galerkin solutions.

Secondly, Conjecture 1 permits us to predict the conditioning of Galerkin matrices for (31). Let us assume that we employ an $\mathbb{L}^{2}(\Sigma)$-orthonormal basis of $\mathrm{H}_{\mathrm{N}}^{h}(\Sigma)$. For these boundary element spaces it takes merely rescaling and local orthogonalisation to build such a basis. Then, again appealing to the $\mathbb{L}^{2}(\Sigma)$-continuity result of Proposition 8 and Conjecture 1 , we can conclude the following:

\section{Proposition 10}

If Conjecture 1 holds true, the Euclidean condition numbers of Galerkin matrices arising from the boundary element discretisation of (31) are bounded from above and below independently of the trial/test space $\mathrm{H}_{\mathrm{N}}^{h}(\Sigma)$, provided that $\mathbb{L}^{2}(\Sigma)$-orthonormal bases are used.

\section{Numerical experiments}

We report two numerical experiments that demonstrate the performance of a Galerkin boundary element discretisation of Formulation (31) for the numerical solution to Problem (1). We concentrate on geometrical configurations featuring junction edges, i.e., edges where at least three subdomains abut. We compare the single-trace second kind Formulation (31) with the so-called direct single-trace first kind approach, described in detail in [9, Section 3], and its Galerkin boundary element discretisation.

For both schemes we rely on conforming, uniformly shape-regular and quasi-uniform skeleton meshes $\mathcal{T}(\Sigma)$ with flat triangular elements. The Galerkin discretisation of (31) is based on piecewise constant discontinuous functions on $\mathcal{T}(\Sigma)$. The same space is used for the approximation of Neumann traces in the first kind STF, whereas for Dirichlet traces we rely on piecewise linear continuous boundary element spaces on $\mathcal{T}(\Sigma)$. Our choice of meshes necessarily involves an approximation of curved interfaces, which should not compromise overall accuracy according to [43, Chapter 8].

All experiments were carried out with the $\mathrm{C}++$ boundary element template library (BETL, [26]). (Nearly) singular integrals were regularised by transformation [43, Chapter 5] and then evaluated by highly accurate numerical quadrature, which ensures that quadrature errors are negligible. The surface meshes were generated using GMSH [20].

\subsection{Experiment I}

In this first experiment we consider a geometrical configuration where space is partitioned in three subdomains $\mathbb{R}^{3}=\bar{\Omega}_{0} \cup \bar{\Omega}_{1} \cup \bar{\Omega}_{2}$, with $\Omega_{1}=\{\boldsymbol{x}=$ $\left(x_{1}, x_{2}, x_{3}\right) \in \mathbb{R}^{3},|\boldsymbol{x}|<1 / 2$ and $\left.x_{3}>0\right\}$, and $\Omega_{2}=\left\{\boldsymbol{x}=\left(x_{1}, x_{2}, x_{3}\right) \in\right.$ $\mathbb{R}^{3},|\boldsymbol{x}|<1 / 2$ and $\left.x_{3}<0\right\}$. The geometry is depicted in Figure 1. Regarding 
the material coefficient $\mu$ we choose the values $\mu_{0}=5, \mu_{1}=1$ and $\mu_{2}=7$, and $u_{\infty}(\boldsymbol{x})=\sin \left(x_{1}\right) \sinh \left(x_{2}\right), \boldsymbol{x}=\left(x_{1}, x_{2}, x_{3}\right) \in \mathbb{R}^{3}$.
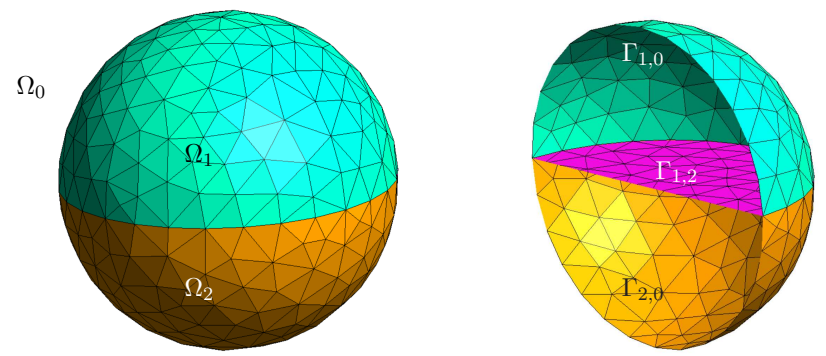

Fig. 1 Experiment I: Geometry

Figure 2 below compares the accuracy of both methods, displaying error norms for Dirichlet or Neumann traces versus meshwidth $h=\max _{\tau \in \mathcal{T}(\Sigma)} \operatorname{diam}(\tau)$. For the computation of the error, the reference solution was taken to be the numerical solution of the second kind STF obtained on an even finer mesh obtained with one additional step of global refinement. We observe algebraic convergence with the same rates and comparable accuracy of both methods.

For these results, the $\mathrm{H}^{-1 / 2}\left(\Gamma_{j}\right)$-norm was approximated using the Galerkin

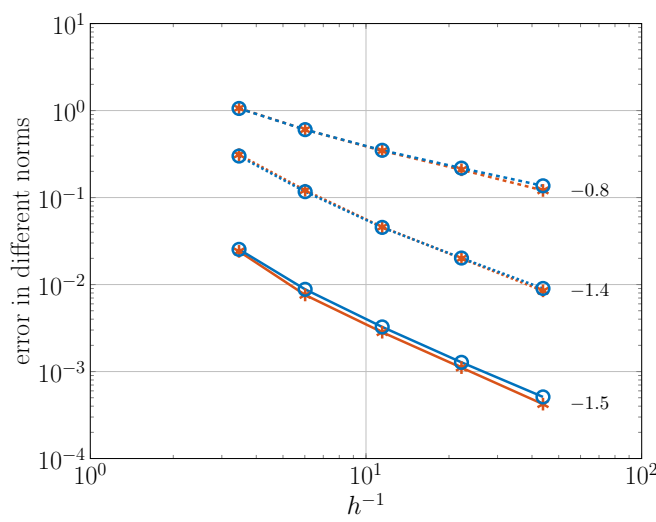

$$
\begin{aligned}
& \text { ——Dirichlet second kind } L^{2}(\Sigma) \\
& \text {---*-- Neumann second kind } L^{2}(\Sigma) \\
& \text {............ Neumann second kind } H^{-1 / 2}(\Sigma) \\
& \text { ○-Dirichlet first kind } L^{2}(\Sigma) \\
& \text {... -... Neumann first kind } L^{2}(\Sigma) \\
& \cdots \cdots \cdots \cdots \cdot \text { Neumann first kind } H^{-1 / 2}(\Sigma)
\end{aligned}
$$

Fig. 2 Experiment I: Convergence of 2nd-kind and 1st-kind STF. The error curves are annotated with estimated convergence rates in terms of $h^{-1}$.

discretisation of the single layer operator $\gamma_{\mathrm{D}}^{j} \cdot \mathrm{SL}_{j}$. Besides, in the case of the second kind formulation, an approximation of the Dirichlet traces of the 
solution was obtained by using the discretised version of the following formula

$$
\gamma_{\mathrm{D}}^{k}(u)=\sum_{j=0}^{n} \gamma_{\mathrm{D}}^{k} \cdot \mathrm{SL}_{j}\left(\gamma_{\mathrm{N}}^{j}(u)\right), \quad k=0 \ldots n .
$$

Figure 3 displays the spectra obtained when solving the generalised eigenvalue problem for the Galerkin matrices and mass matrices. Here and in the sequel, $\mathcal{N}_{\mathrm{T}}$ refers to the number of triangles of the mesh. They can be viewed as approximations of the spectrum of the continuous operators. While in the case of the first kind STF many eigenvalues cluster in a neighbourhood of 0 , in the case of the second kind STF the eigenvalues remain nicely separated from the origin.
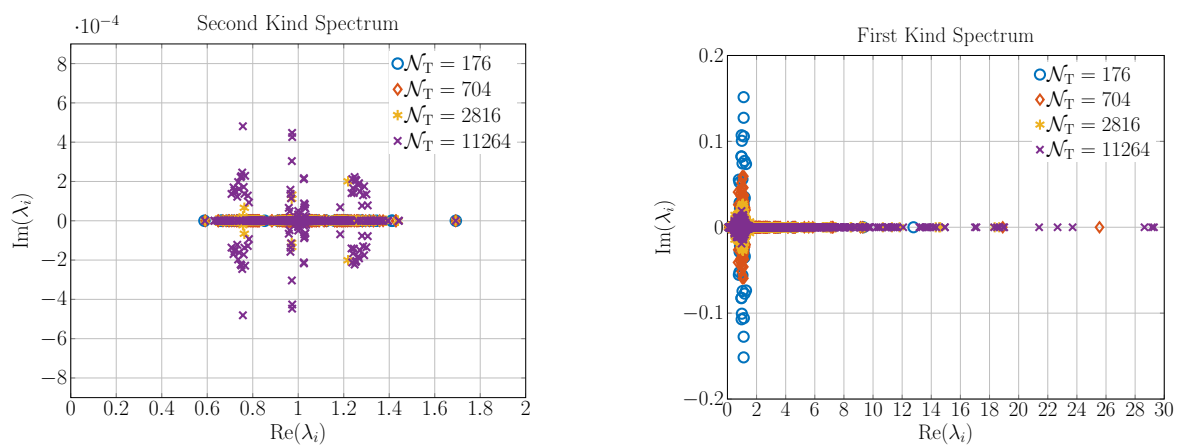

Fig. 3 Experiment I: Spectrum of Galerkin matrices for 2nd-kind (left) and 1st-kind (right) $\mathrm{STF}$

This suggests good convergence of linear iterative solvers applied to the second kind STF, which is confirmed by the plots of Figure 4. The Galerkin matrices for the second kind STF enjoy much better conditioning, and the condition numbers remain stable with respect to the meshwidth, while the condition numbers for the first kind STF deteriorate as $h \rightarrow 0$. This is reflected by the behaviour of GMRES iterations.

\subsection{Experiment II}

Now we consider a partition of space with one more subdomain $\mathbb{R}^{3}=\bar{\Omega}_{0} \cup$ $\bar{\Omega}_{1} \cup \bar{\Omega}_{2} \cup \bar{\Omega}_{3}$, with $\Omega_{1}=\left\{\boldsymbol{x}=\left(x_{1}, x_{2}, x_{3}\right) \in \mathbb{R}^{3},|\boldsymbol{x}|<1 / 2\right.$ and $\left.x_{3}>0\right\}, \Omega_{2}=$ $\left\{\boldsymbol{x}=\left(x_{1}, x_{2}, x_{3}\right) \in \mathbb{R}^{3},|\boldsymbol{x}|<1 / 2\right.$ and $\left.x_{3}<0\right\}$, and $\Omega_{3}=\mathcal{Q} \backslash \bar{\Omega}_{2}$ with $\mathcal{Q}:=$ $(-0.7,+0.7) \times(-0.7,+0.7) \times(0,0.7)$. We choose $\mu_{0}=5, \mu_{1}=1, \mu_{2}=7$ and $\mu_{3}=3$. The excitation field is the same as before $u_{\infty}(\boldsymbol{x})=\sin \left(x_{1}\right) \sinh \left(x_{2}\right)$, $\boldsymbol{x}=\left(x_{1}, x_{2}, x_{3}\right) \in \mathbb{R}^{3}$. The geometry is represented in Figure 5 .

We report the same quantities as in Experiment I and make the same observations in Figures 10.2-10.2: The accuracy of both methods is comparable, 

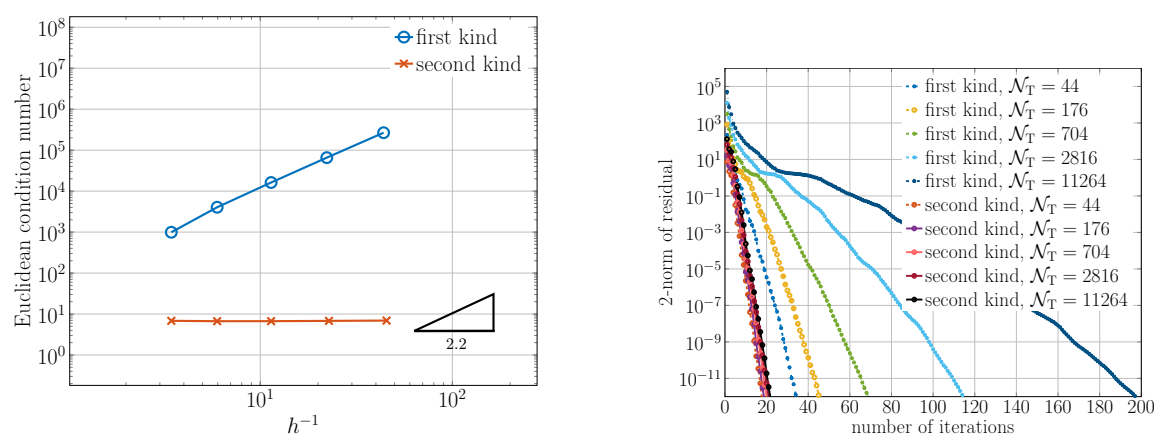

Fig. 4 Experiment I: Euclidean condition numbers (left) and convergence history of GMRES in terms of decrease of Euclidean norm of residual vectors (right)
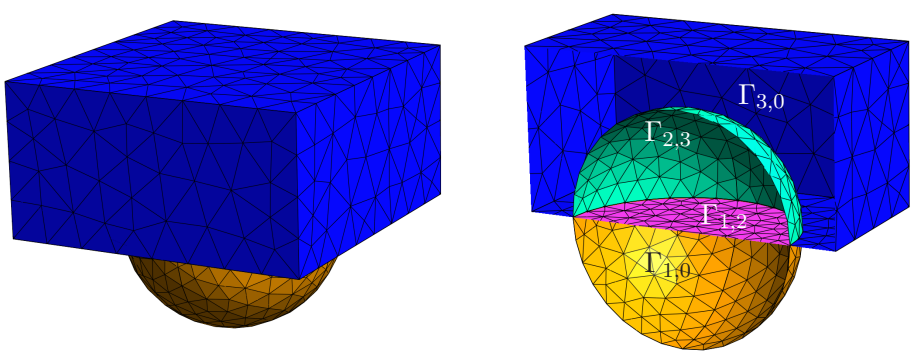

Fig. 5 Experiment II: Geometric setup

while the second kind STF leads to matrices much lower condition numbers, which translates into much faster convergence of GMRES.
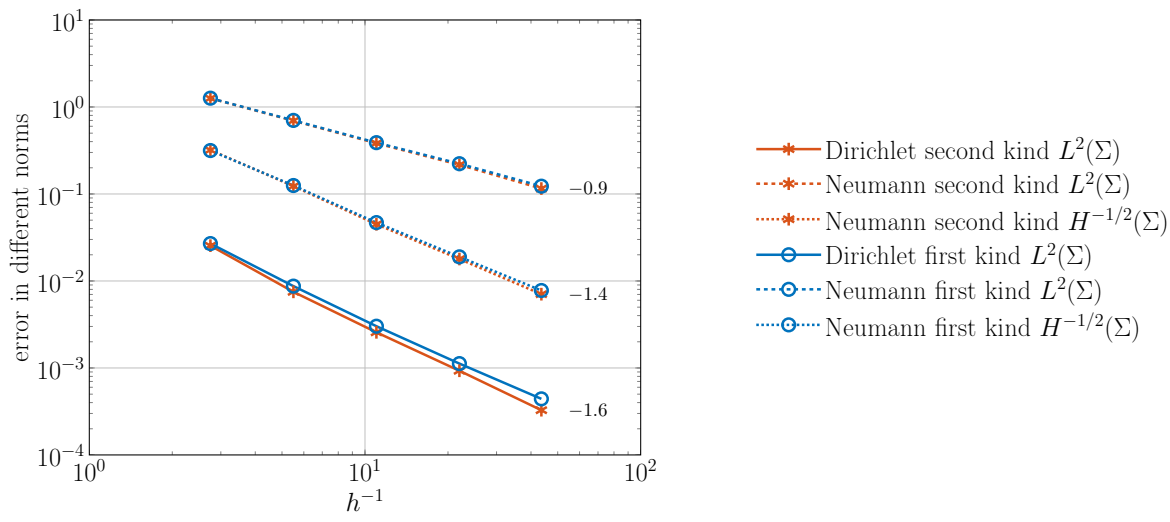

Fig. 6 Experiment II, $c f$. Figure 2 

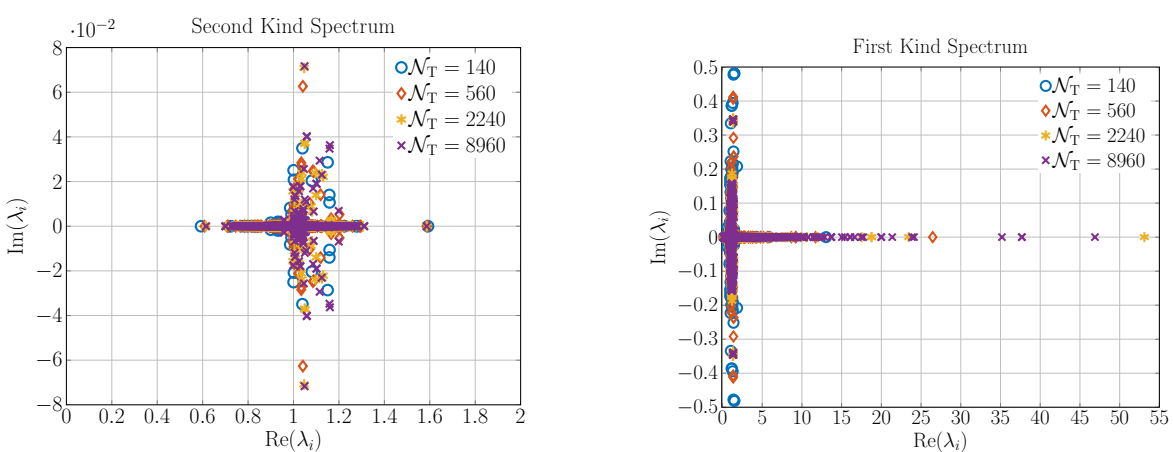

Fig. 7 Experiment II, $c f$. Figure 3
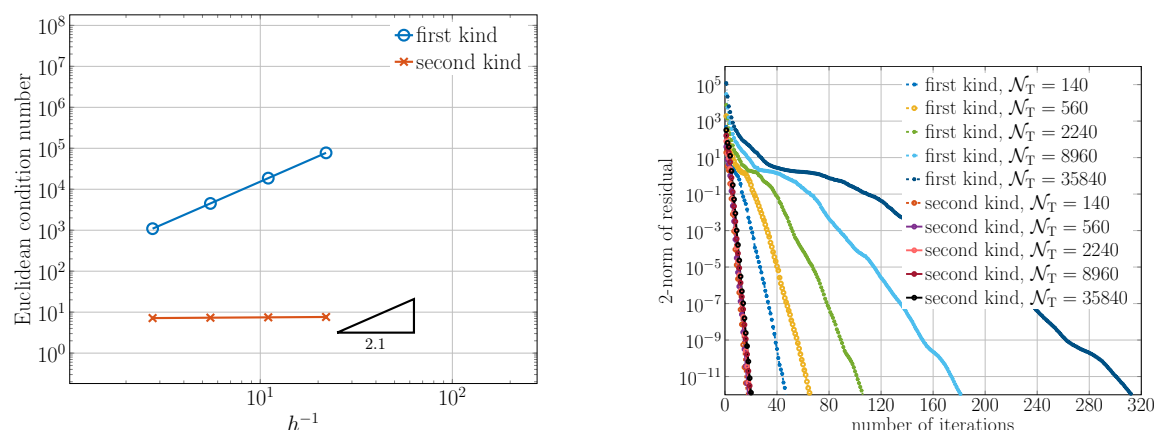

Fig. 8 Experiment II, $c f$. Figure 4

\section{Conclusion}

We derived and analysed a novel second kind single-trace boundary integral equation formulation for 2nd-order diffusion transmission problems with piecewise constant scalar diffusion coefficients. The unknown is a single function on the skeleton, representing the jump of normal flux traces across interfaces. Well-posedness of the BIE in low-regularity Sobolev spaces could be established. In numerical tests boundary element Galerkin discretisation led to wellconditioned linear systems and yielded satisfactory approximate solutions, but its numerical analysis remains wide open.

Acknowledgements The work of X.Claeys received support from the ANR research grant ANR-15-CE23-0017-01. E. Spindler was supported by SNF grant 200021_137873/1.

\section{References}

1. Agranovich, M.S.: Sobolev spaces, their generalizations and elliptic problems in smooth and Lipschitz domains. Springer Monographs in Mathematics. Springer, Cham (2015) 
2. Bonito, A., Guermond, J.L., Luddens, F.: Regularity of the Maxwell equations in heterogeneous media and Lipschitz domains. J. Math. Anal. Appl. 408(2), 498-512 (2013)

3. Buffa, A.: Remarks on the discretization of some noncoercive operator with applications to the heterogeneous Maxwell equations. SIAM J. Numer. Anal. 43(1), 1-18 (2005)

4. Buffa, A., Hiptmair, R.: Galerkin boundary element methods for electromagnetic scattering. In: Topics in computational wave propagation, Lect. Notes Comput. Sci. Eng., vol. 31, pp. 83-124. Springer, Berlin (2003)

5. Chang, Y., Harrington, R.: A surface formulation or characteristic modes of material bodies. IEEE Trans. Antennas and Propagation 25, 789-795 (1977)

6. Claeys, X.: A single trace integral formulation of the second kind for acoustic scattering. Tech. Rep. no. 2011-14, SAM, ETH Zürich (2011)

7. Claeys, X., Hiptmair, R.: Electromagnetic scattering at composite objects : a novel multi-trace boundary integral formulation. ESAIM: Mathematical Modelling and Numerical Analysis 46, 1421-1445 (2012)

8. Claeys, X., Hiptmair, R.: Multi-trace boundary integral formulation for acoustic scattering by composite structures. Comm. Pure Appl. Math. 66(8), 1163-1201 (2013)

9. Claeys, X., Hiptmair, R., Jerez-Hanckes, C.: Multi-trace boundary integral equations. In: Direct and Inverse Problems in Wave Propagation and Applications. I. Graham, U. Langer, M. Sini, M. Melenk (2012)

10. Claeys, X., Hiptmair, R., Jerez-Hanckes, C., Pintarelli, S.: Novel multitrace boundary integral equations for transmission boundary value problems. In: Unified transform for boundary value problems, pp. 227-258. SIAM, Philadelphia, PA (2015)

11. Claeys, X., Hiptmair, R., Spindler, E.: Second-kind boundary integral equations for scattering at composite partly impenetrable objects. Tech. Rep. 2015-19, Seminar for Applied Mathematics, ETH Zürich, Switzerland (2015)

12. Claeys, X., Hiptmair, R., Spindler, E.: A second-kind Galerkin boundary element method for scattering at composite objects. BIT 55(1), 33-57 (2015)

13. Claeys, X., Hiptmair, R., Spindler, E.: Second-kind boundary integral equations for electromagnetic scattering at composite objects. Preprint 2016-43, SAM, ETH Zurich, Switzerland (2016)

14. Coifman, R., Jones, P., Semmes, S.: Two elementary proofs of the $L^{2}$ boundedness of Cauchy integrals on Lipschitz curves. J. Amer. Math. Soc. 2(3), 553-564 (1989)

15. Coifman, R.R., McIntosh, A., Meyer, Y.: L’intégrale de Cauchy définit un opérateur borné sur $L^{2}$ pour les courbes lipschitziennes. Ann. of Math. (2) 116(2), 361-387 (1982)

16. Costabel, M.: Boundary integral operators on Lipschitz domains: elementary results. SIAM J. Math. Anal. 19(3), 613-626 (1988)

17. Costabel, M., Stephan, E.: A direct boundary integral equation method for transmission problems. J. Math. Anal. Appl. 106(2), 367-413 (1985). DOI 10.1016/0022247X(85)90118-0. URL http://dx.doi.org/10.1016/0022-247X(85)90118-0

18. Dahlberg, B.: Estimates of harmonic measure. Arch. Rational Mech. Anal. 65(3), 275288 (1977)

19. Dahlberg, B.: On the Poisson integral for Lipschitz and $C^{1}$-domains. Studia Math. 66(1), 13-24 (1979)

20. Geuzaine, C., Remacle, J.F.: Gmsh: A 3-d finite element mesh generator with builtin pre- and post-processing facilities. International Journal for Numerical Methods in Engineering 79(11), 1309-1331 (2009)

21. Greengard, L., Lee, J.Y.: Stable and accurate integral equation methods for scattering problems with multiple material interfaces in two dimensions. J. Comput. Phys. 231(6), 2389-2395 (2012)

22. Hackbusch, W.: Integral equations, International Series of Numerical Mathematics, vol. 120. Birkhäuser Verlag, Basel (1995). Theory and numerical treatment, Translated and revised by the author from the 1989 German original

23. Hardy, G.H., Littlewood, J.E., Pólya, G.: Inequalities. Cambridge, at the University Press (1952). 2d ed

24. Harrington, R.: Boundary integral formulations for homogeneous material bodies. Journal of Electromagnetic Waves and Applications 3(1), 1-15 (1989) 
25. Hiptmair, R., Jerez-Hanckes, C.: Multiple traces boundary integral formulation for Helmholtz transmission problems. Adv. Comput. Math. 37(1), 39-91 (2012). DOI 10.1007/s10444-011-9194-3. URL http://dx.doi.org/10.1007/s10444-011-9194-3

26. Hiptmair, R., Kielhorn, L.: Betl a generic boundary element template library. Tech. Rep. 2012-36, Seminar for Applied Mathematics, ETH Zürich, Switzerland (2012)

27. Hsiao, G.C., Steinbach, O., Wendland, W.L.: Domain decomposition methods via boundary integral equations. J. Comput. Appl. Math. 125(1-2), 521-537 (2000). DOI 10.1016/S0377-0427(00)00488-X. URL http://dx.doi.org/10.1016/S03770427(00)00488-X. Numerical analysis 2000, Vol. VI, Ordinary differential equations and integral equations

28. Kleinmann, R., Martin, P.: On single integral equations for the transmission problem of acoustics. SIAM J. Appl. Math. 48(2), 307-325 (1988)

29. Langer, U., Steinbach, O.: Boundary Element Tearing and Interconnecting Methods. Computing 71, 205-228 (2003)

30. McLean, W.: Strongly elliptic systems and boundary integral equations. Cambridge University Press, Cambridge (2000)

31. Mercier, D.: Minimal regularity of the solutions of some transmission problems. Math. Methods Appl. Sci. 26(4), 321-348 (2003)

32. Miller, E., Poggio, A.: Computer Techniques for Electromagnetics, vol. 7, chap. Chap. 4, Integral equation solutions of three-dimensional scattering problems. Pergamon Press (1973)

33. Nicaise, S., Sändig, A.M.: General interface problems. I, II. Math. Methods Appl. Sci. 17(6), 395-429, 431-450 (1994)

34. Of, G., Steinbach, O.: The all-floating boundary element tearing and interconnecting method. J. Numer. Math. 17(4), 277-298 (2009)

35. Of, G., Steinbach, O., Wendland, W.L.: Boundary element tearing and interconnecting domain decomposition methods. In: Multifield problems in solid and fluid mechanics, Lect. Notes Appl. Comput. Mech., vol. 28, pp. 461-490. Springer, Berlin (2006)

36. Opic, B., Kufner, A.: Hardy-type inequalities, Pitman Research Notes in Mathematics Series, vol. 219. Longman Scientific \& Technical, Harlow (1990)

37. Peng, Z., Lim, K.H., Lee, J.F.: Computations of electromagnetic wave scattering from penetrable composite targets using a surface integral equation method with multiple traces. IEEE Trans. Antennas and Propagation 61(1), 256-270 (2013)

38. Peng, Z., Lim, K.H., Lee, J.F.: A boundary integral equation domain decomposition method for electromagnetic scattering from large and deep cavities. J. Comput. Phys. 280, 626-642 (2015)

39. Petersdorff, T.v.: Boundary Integral Equations for Mixed Dirichlet, Neumann and Transmission Problems. Math. Met. App. Sc. 11, 185-213 (1989)

40. Petzoldt, M.: Regularity results for Laplace interface problems in two dimensions. Z. Anal. Anwendungen 20(2), 431-455 (2001)

41. Rudin, W.: Real and complex analysis, third edn. McGraw-Hill Book Co., New York (1987)

42. Rumsey, V.H.: Reaction concept in electromagnetic theory. Phys. Rev. 94, 1483-1491 (1954)

43. Sauter, S., Schwab, C.: Boundary element methods, Springer Series in Computational Mathematics, vol. 39. Springer-Verlag, Berlin (2011)

44. Savaré, G.: Regularity results for elliptic equations in Lipschitz domains. J. Funct. Anal. 152(1), 176-201 (1998)

45. Spindler, E.: Second kind single-trace boundary integral formulations for scattering at composite objects. Ph.D. thesis, Seminar of Applied Mathematics, ETH Zürich (2016). Diss. no 23579, Prof. Dr. Ralf Hiptmair

46. Verchota, G.: Layer potentials and regularity for the Dirichlet problem for Laplace's equation in Lipschitz domains. J. Funct. Anal. 59(3), 572-611 (1984) 